\title{
RELAÇÕES FUNCIONAIS ENTRE PROPRIEDADES FÍSICAS E MECÂNICAS DE MADEIRAS TROPICAIS BRASILEIRAS
}

\author{
Henrique José Borges de Araújo* \\ *Eng. Florestal, M.Sc., Embrapa - henrique@cpafac.embrapa.br \\ Recebido para publicação: 20/03/2007 - Aceito para publicação: 03/05/2007
}

\begin{abstract}
Resumo
Este estudo foi realizado com o objetivo de avaliar as relações funcionais entre as propriedades físicas e mecânicas de 163 espécies de madeiras tropicais brasileiras e, também, oferecer recursos matemáticos que permitam estimá-las. Foram efetuadas avaliações das correlações e análises de regressão, sendo que os dados da amostra foram obtidos pelas normas de ensaio da COPANT. As propriedades utilizadas foram: densidade básica $\left(\rho_{\text {bas }}\right)$, contração tangencial $\left(\varepsilon_{\mathrm{r}, 3}\right)$ e radial $\left(\varepsilon_{\mathrm{r}, 2}\right)$, dureza Janka paralela $\left(f_{\mathrm{H} 0}\right)$ e transversal $\left(\mathrm{f}_{\mathrm{H} 90}\right)$ às fibras, e as relativas à resistência a esforços mecânicos de compressão $\left(\mathrm{f}_{\mathrm{c} 0}\right.$ e $\left.\mathrm{E}_{\mathrm{c} 90}\right)$, flexão estática $\left(f_{M}\right.$ e $\left.E_{M 0}\right)$, tração $\left(f_{w 190}\right)$, fendilhamento $\left(f_{v 0}\right)$ e cisalhamento $\left(f_{s 0}\right)$. As correlações foram expressas pelo coeficiente de correlação linear de Pearson, e nas análises de regressão utilizou-se $\rho_{\text {bas }}$ como variável independente. Foram realizadas análises das suposições estatísticas para ajuste das equações de regressão. As propriedades com as mais altas relações funcionais foram $f_{c 0}, f_{H 90}, \rho_{\text {bas }}, f_{\mathrm{M}}$, $\mathrm{f}_{\mathrm{H} 0}, \mathrm{E}_{\mathrm{M} 0}, \mathrm{f}_{\mathrm{s} 0}$ e $\mathrm{E}_{\mathrm{c} 90}$, e as com as mais baixas foram $\varepsilon_{\mathrm{r}, 3}, \varepsilon_{\mathrm{r}, 2}, \mathrm{f}_{\mathrm{w} t 90}$ e $\mathrm{f}_{\mathrm{v} 0}$. As análises de regressão possibilitaram a obtenção de equações satisfatórias para estimativas das propriedades físicas e mecânicas de madeiras de espécies brasileiras com as mais altas relações funcionais.

Palavras-chave: Madeira tropical; propriedades físicas da madeira; propriedades mecânicas da madeira; madeira; propriedades da madeira.
\end{abstract}

\begin{abstract}
Functional relationships among physical and mechanical properties of Brazilian tropical woods. This study was done with the objective of evaluating the functional relationships among the physical and mechanical properties of 163 species of Brazilian tropical woods, and also to offer mathematical tools that allow the estimation of them. Evaluations of correlations and of regression analysis were done, and the data of the samples were obtained according to the COPANT standards. The properties used were: basic density $\left(\rho_{\text {bas }}\right)$, tangential $\left(\varepsilon_{\mathrm{r}, 3}\right)$ and radial $\left(\varepsilon_{\mathrm{r}, 2}\right)$ shrinkages, Janka hardness parallel $\left(\mathrm{f}_{\mathrm{H} 0}\right)$ and crossection $\left(f_{\mathrm{H} 90}\right)$ to the grain, and those properties related to the resistance to mechanical efforts of compression $\left(\mathrm{f}_{\mathrm{c} 0}\right.$ and $\left.\mathrm{E}_{\mathrm{c} 90}\right)$, static bending $\left(\mathrm{f}_{\mathrm{M}}\right.$ and $\left.\mathrm{E}_{\mathrm{M} 0}\right)$, traction $\left(\mathrm{f}_{\mathrm{w} t 90}\right)$, cleavage $\left(\mathrm{f}_{\mathrm{v} 0}\right)$ and shear $\left(\mathrm{f}_{\mathrm{s} 0}\right)$. The correlations were expressed by the coefficient of linear correlation of Pearson and in the regression analysis $\rho_{\text {bas }}$ was used as independent variable. Analysis of the statistical suppositions were carried out in order to do adjustments of the regression equations. The properties with the highest functional relationships were $\mathrm{f}_{\mathrm{c} 0}, \mathrm{f}_{\mathrm{H} 90}, \rho_{\mathrm{bas}}, \mathrm{f}_{\mathrm{M}}, \mathrm{f}_{\mathrm{H} 0}, \mathrm{E}_{\mathrm{M} 0}, \mathrm{f}_{\mathrm{s} 0}$ and $\mathrm{E}_{\mathrm{c} 90}$ and the lowest were $\varepsilon_{\mathrm{r}, 3}, \varepsilon_{\mathrm{r}, 2}, \mathrm{f}_{\mathrm{wt} 90}$ and $\mathrm{f}_{\mathrm{v} 0}$. The regression analysis made possible the obtaition of satisfactory equations for estimates of the physical and mechanical properties of Brazilian tropical wood species with the highest functional relationships. Keywords: Tropical wood; physical properties; mechanical properties; wood; properties.
\end{abstract}

\section{INTRODUÇ̃̃̃O}

O conhecimento das propriedades físicas e mecânicas das madeiras (PFMM) é fundamental para definir adequadamente as aplicações a que serão destinadas e dimensionar, com segurança, as partes componentes de uma estrutura com esse material. Combinados a outros fatores (resistência à degradação biológica e abiótica, facilidade de processamento com ferramentas, valor econômico de mercado, estético, etc.), os parâmetros dessas propriedades permitem que as madeiras sejam classificadas em usos a que se mostram mais apropriadas, como, por exemplo, peças estruturais, ambientes internos e externos de habitações, mobílias, painéis e embalagens. 
Relativamente às PFMM, a indicação de uma madeira para determinada aplicação considera atributos referentes ao seu peso, ao comportamento frente às condições ambientais (umidade, principalmente) e, também, aos limites de resistência mecânica correspondentes aos esforços a que estarão sujeitas nas situações de uso.

A mensuração das PFMM é efetuada através de ensaios de laboratório, utilizando equipamentos próprios a essa finalidade e seguindo normas que especificam os métodos, procedimentos, fórmulas de cálculo, formas e dimensões de corpos de prova, etc. As normas de ensaio mais empregadas mundialmente são as da American Society for Testing and Materials (ASTM), as da British Standard Institution (BSI), as da International Organization for Standardization (ISO) e as da Comisión Panamericana de Normas Técnicas (COPANT). No Brasil, existem as normas da Associação Brasileira de Normas Técnicas (ABNT).

Entre as principais propriedades físicas da madeira estão a massa específica (ou densidade), expressa pelo quociente entre a massa e o volume, e a instabilidade dimensional, representada pela variação das dimensões (linear e volumétrica) em função do teor de umidade. Dentre as propriedades mecânicas, destacam-se a resistência à ação de forças externas, tais como compressão, flexão, tração, cisalhamento e fendilhamento.

Muito embora já se tenha ensaiado um grande número de espécies de madeira, ainda restam muitas a serem estudadas. Araújo (2002), ao realizar extenso levantamento na literatura nacional e internacional acerca das PFMM de 197 espécies tropicais ocorrentes em uma área florestal no estado do Acre, na região Amazônica, encontrou o nome científico completo (gênero e espécie) de apenas 74 delas (37,5\%), sendo que 113 espécies (57,4\%) foram localizadas somente no nível de gênero, e $10(5,1 \%)$ não foram encontradas na literatura. O levantamento de Araújo (2002) revelou uma significativa lacuna em pesquisas sobre madeiras amazônicas.

A fim de avaliar as relações funcionais existentes entre as PFMM de espécies tropicais brasileiras, procedeu-se o estudo de suas correlações e de suas dependências funcionais (análises de regressão). Este estudo, baseado em uma amostra de dados de 163 espécies, tem como objetivo conhecer o grau de correspondência entre essas propriedades e, ao mesmo tempo, oferecer recursos matemáticos (equações de regressão) que possibilitem estimativas para espécies ainda não estudadas.

\section{REVISÃO BIBLIOGRÁFICA}

Entre as PFMM, a massa específica (densidade) é a que mais se destaca. Essa propriedade tem relação direta com a composição química da madeira e revela a quantidade do material lenhoso da parede celular, estando, dessa maneira, estreitamente relacionada com outras propriedades (NAHUZ, 1974; RICHTER; BURGER, 1978). Devido à variação nas dimensões e proporção dos diversos tecidos da madeira, a massa específica pode variar de 0,13 a $1,40 \mathrm{~g} . \mathrm{cm}^{-3}$ entre as espécies, no entanto, a massa específica da matéria lenhosa sólida varia pouco, podendo-se admitir um valor médio de 1,50 g. $\mathrm{cm}^{-3}$ para todas as madeiras (KOLLMANN; CÔTÉ, 1968).

Neste trabalho, a massa específica é representada pela densidade básica. Segundo Lopes e Garcia (2002), a densidade básica da madeira é definida pela relação entre seu peso absolutamente seco e seu volume saturado (umidade acima do Ponto de Saturação das Fibras - PSF) e expressa em g.cm ${ }^{-3}$.

A densidade básica é de fácil obtenção e um dos principais parâmetros para aferir a qualidade da madeira, sendo um excelente índice para indicar seu emprego nas diversas finalidades (PANSHIN; ZEEUW, 1970). Em termos gerais, pode-se dizer que as variações da densidade básica entre espécies estão relacionadas às suas características anatômicas, como, por exemplo, o comprimento e largura da célula, espessura da parede celular, diâmetro do lume e proporção e distribuição dos tecidos no lenho (LOPES; GARCIA, 2002).

Como material estrutural, a madeira possui a desvantagem de ser heterogênea. Sequer amostras de uma mesma árvore apresentam valores das PFMM absolutamente iguais (BROWN et al., 1949). Segundo Rocha (1994), a heterogeneidade da madeira se deve aos diversos tipos de células com funções específicas, ao fato de ser constituída de uma série de compostos químicos, orgânicos e inorgânicos, e também aos fatores que afetam o desenvolvimento das árvores, tais como clima, solo, local de crescimento e os de ordem genética.

Ainda que as PFMM tenham estreita relação com a massa específica, há outros importantes fatores de influência. O comportamento dimensional e a resistência a esforços mecânicos da madeira, além da dependência da quantidade de material celulósico na parede celular, relacionam-se com as 
proporções e arranjos dos seus componentes estruturais anatômicos (fibrilar, parenquimatosos, etc.) e aos elementos não-estruturais, como extrativos e água (PANSHIN; ZEEUW, 1970; WANGAARD, 1950).

Arganbright (1971), avaliando a influência de extrativos nas propriedades da madeira de uma conífera norte-americana, constatou que a quantidade de extrativos é diretamente proporcional à resistência aos esforços mecânicos de compressão paralela às fibras e à resistência ao choque, porém, não altera os valores do módulo de ruptura à flexão estática e é inversamente proporcional aos valores do módulo de elasticidade à flexão estática. Garcia e Quirino (1993) constataram que a remoção de apenas 1,9\% de extrativos da madeira de ipê (Tabebuia sp) ocasionou um decréscimo de $17,3 \%$ na resistência de ruptura à compressão paralela às fibras.

A presença da água afeta sobremaneira a resistência da madeira. As variações na umidade produzem diferenças na massa especifica, resultando, portanto, em variações na resistência aos esforços mecânicos. Segundo Panshin e Zeeuw (1970), a resistência mecânica se altera inversamente com o conteúdo de umidade abaixo do PSF. Acima do PSF, têm-se evidenciado na literatura que as propriedades mecânicas da madeira permanecem estáveis quando ocorrem variações da umidade.

Além dos fatores mencionados, ainda há outros relevantes que influenciam as PFMM, podendose citar as proporções dos elementos da parede celular (celulose, hemiceluloses e lignina), a disposição dos vasos e porosidade, a posição da amostra no tronco da árvore, a direção das fibras (longitudinal e transversal) onde são aplicadas as forças externas e a idade da árvore (KOLLMANN; CÔTÉ, 1968; PANSHIN; ZEEUW, 1970; FOREST, 1974; RICHTER; BURGER, 1978; JANKOWSKY, 1979; SIAU, 1984; ROCHA et al., 1988; GARCIA; QUIRINO, 1993; EVANS et al., 2000).

Embora concisas, as informações bibliográficas apresentadas assinalam algumas particularidades fundamentais para melhor compreensão das madeiras em relação às PFMM. A primeira é de que é possível admitir que a massa específica é o melhor indicativo para definição dos usos condicionados à resistência a forças mecânicas, uma vez que a grande maioria das propriedades mecânicas está firmemente associada a essa propriedade. Além disso, a obtenção da massa específica não requer complexas e onerosas máquinas de ensaio e, desse modo, torna-se importante também sob o ponto de vista econômico, considerando-se que seus custos de ensaio em laboratório são reduzidos. Outro aspecto relevante diz respeito à influência da umidade. Na maior parte das situações de uso, a umidade do ar é um fator não controlável e inconstante e, em razão da higroscopicidade, a madeira tende ao equilíbrio com o ambiente, estando sujeita, portanto, a modificar com freqüência os valores que as PFMM podem assumir.

\section{MATERIAL E MÉTODOS}

\section{Propriedades físicas e mecânicas utilizadas}

Para o presente estudo, foram utilizadas doze PFMM, sendo três físicas e nove mecânicas (Tabela 1).

Tabela 1. Propriedades físicas e mecânicas das madeiras (PFMM) utilizadas no estudo.

Table 1. Physical and mechanical properties of wood (PFMM) used in the study.

\begin{tabular}{|c|c|c|c|c|c|}
\hline \multicolumn{3}{|c|}{ Propriedade } & \multirow{2}{*}{$\frac{\text { Notação }^{1}}{\rho_{\text {bas }}}$} & \multirow{2}{*}{$\begin{array}{c}\begin{array}{c}\text { Unidade de } \\
\text { medida }\end{array} \\
\mathrm{g} \cdot \mathrm{cm}^{-3}\end{array}$} & \multirow{2}{*}{$\begin{array}{c}\begin{array}{c}\text { Teor de } \\
\text { umidade }^{2}\end{array} \\
-\end{array}$} \\
\hline Físicas & 1. & Densidade básica & & & \\
\hline \multirow{11}{*}{ Mecânicas } & 2. & Contração tangencial & $\varepsilon_{\mathrm{r}, 3}$ & $\%$ & - \\
\hline & & Contração radial & $\varepsilon_{\mathrm{r}, 2}$ & $\%$ & - \\
\hline & 4. & Módulo de ruptura à flexão estática & $\mathrm{f}_{\mathrm{M}}$ & MPa & $12 \%$ \\
\hline & & Módulo de elasticidade à flexão estática & $\mathrm{E}_{\mathrm{M} 0}$ & MPa & $12 \%$ \\
\hline & & Resistência à compressão paralela às fibras & $\mathrm{f}_{\mathrm{c} 0}$ & $\mathrm{MPa}$ & $12 \%$ \\
\hline & 7. & $\begin{array}{l}\text { Resistência no limite proporcional à compressão } \\
\text { perpendicular às fibras }\end{array}$ & $\mathrm{E}_{\mathrm{c} 90}$ & $\mathrm{MPa}$ & $12 \%$ \\
\hline & & Dureza Janka paralela às fibras & $\mathrm{f}_{\mathrm{H} 0}$ & $\mathrm{~N}$ & $12 \%$ \\
\hline & & Dureza Janka transversal às fibras & $\mathrm{f}_{\mathrm{H} 90}$ & $\mathrm{~N}$ & $12 \%$ \\
\hline & 10. & Resistência à tração perpendicular às fibras & $\mathrm{f}_{\mathrm{wt} 90}$ & $\mathrm{MPa}$ & $12 \%$ \\
\hline & 11. & Resistência ao fendilhamento & $\mathrm{f}_{\mathrm{v} 0}$ & $\mathrm{MPa}$ & $12 \%$ \\
\hline & 12. & Resistência ao cisalhamento & $\mathrm{f}_{\mathrm{s} 0}$ & $\mathrm{MPa}$ & $12 \%$ \\
\hline
\end{tabular}

MPa: MegaPascal; N: Newton; ${ }^{1}$ : conforme norma NBR 7190 (ABNT); ${ }^{2}$ : refere-se ao teor de umidade do corpo de prova do respectivo ensaio. 
As definições sumárias e as fórmulas de cálculo de cada PFMM são apresentadas na tabela 2. Tais informações foram adaptadas da norma NBR 7190 (ASSOCIAÇÃO, 1997).

Tabela 2. Propriedades físicas e mecânicas utilizadas no estudo (adaptado de ASSOCIAÇÃO, 1997).

Table 2. Physical and mechanical properties used in the study (adapted of ASSOCIAÇÃO, 1997).

\begin{tabular}{|c|c|c|c|c|}
\hline Propriedade & Notação & Descrição sumária & Fórmula de cálculo & Componentes da fórmula \\
\hline $\begin{array}{l}\text { 1. Densidade } \\
\text { básica }\end{array}$ & $\rho_{\text {bas }}$ & $\begin{array}{l}\text { É uma massa específica convencional da } \\
\text { madeira dada pelo quociente entre a } \\
\text { massa seca em estufa (teor de umidade } \\
\text { de } 0 \% \text { e o volume verde (teor de } \\
\text { umidade saturado). }\end{array}$ & $\rho_{\text {bas }}=\frac{M s}{V v}$ & $\begin{array}{l}\rho_{\text {bas }}=\text { densidade básica, em } \mathrm{g} . \mathrm{cm}^{-3} . \\
M s=\text { massa seca em estufa, em } \mathrm{g}, \text { ao t.u. de } \\
0 \% . \\
V v=\text { volume verde, } \mathrm{em} \mathrm{cm}^{3} \text {, ao t.u. saturado. }\end{array}$ \\
\hline $\begin{array}{l}\text { 2. Contração } \\
\text { tangencial }\end{array}$ & $\varepsilon_{\mathrm{r}, 3}$ & $\begin{array}{l}\text { É a retração da dimensão da madeira na } \\
\text { direção tangencial aos anéis de } \\
\text { crescimento, ou na direção perpendicular } \\
\text { aos raios, que ocorre quando o teor de } \\
\text { umidade passa do saturado para } 0 \% \text { seco } \\
\text { em estufa. }\end{array}$ & $\varepsilon_{\mathrm{r}, 3}=\left(\frac{\mathrm{Lt}_{\mathrm{sat}}-\mathrm{Lt}_{0 \%}}{\mathrm{Lt}_{\mathrm{sat}}}\right) 100$ & $\begin{array}{l}\varepsilon_{\mathrm{r}, 3}=\text { contração tangencial, em } \% \text {. } \\
L t_{s a t}=\text { dimensão da direção tangencial aos } \\
\text { anéis de crescimento, em cm, ao t.u. saturado } \\
L t_{0 \%}=\text { dimensão da direção tangencial aos } \\
\text { anéis de crescimento, em cm, ao t.u. de } 0 \% \text {. }\end{array}$ \\
\hline $\begin{array}{l}\text { 3. Contração } \\
\text { radial }\end{array}$ & $\varepsilon_{\mathrm{r}, 2}$ & $\begin{array}{l}\text { E a retração da dimensão da madeira na } \\
\text { direção longitudinal aos raios, ou na } \\
\text { direção perpendicular aos anéis de } \\
\text { crescimento, que ocorre quando o teor de } \\
\text { umidade passa do saturado para } 0 \% \text { seco } \\
\text { em estufa. }\end{array}$ & $\varepsilon_{\mathrm{r}, 2}=\left(\frac{\mathrm{Lr}_{\mathrm{sat}}-\mathrm{Lr}_{0 \%}}{\mathrm{Lr}_{\mathrm{sat}}}\right) 100$ & $\begin{array}{l}\varepsilon_{\mathrm{r}, 2}=\text { contração radial, em \% } \\
L r_{\text {sat }}=\text { dimensão da direção longitudinal aos } \\
\text { raios, em cm, ao t.u. saturado } \\
L r_{0 \%}=\text { dimensão da direção longitudinal aos } \\
\text { raios, em cm, ao t.u. de } 0 \%\end{array}$ \\
\hline $\begin{array}{l}\text { 4. Módulo de } \\
\text { ruptura à flexão } \\
\text { estática }\end{array}$ & $f_{M}$ & $\begin{array}{l}\text { É a resistência à ruptura da madeira à } \\
\text { flexão estática dada pelo quociente entre } \\
\text { o máximo momento que pode atuar em } \\
\text { um corpo de prova e o módulo de } \\
\text { resistência elástico da seção transversal } \\
\text { (dada por b.h } h^{2} \cdot 6^{-1} \text {, onde b e h são os } \\
\text { lados) desse corpo de prova. É calculado } \\
\text { com a hipótese da madeira ser um } \\
\text { material elástico. }\end{array}$ & $\mathrm{f}_{\mathrm{M}}=\frac{\mathrm{M}_{\max }}{\mathrm{We}}$ & $\begin{array}{l}\mathrm{f}_{\mathrm{M}}=\text { módulo de ruptura à flexão estática, em } \\
\mathrm{N} . \mathrm{m}^{-2} \text {, ou } \mathrm{MPa} \text {, ao t.u. de } 12 \% \text {. } \\
M_{\max }=\text { máximo momento, em } \mathrm{Nm} \text {, atuante no } \\
\text { corpo de prova. } \\
W e=\text { módulo de resistência elástico, } \mathrm{em} \mathrm{m}^{3} \text {. }\end{array}$ \\
\hline $\begin{array}{l}\text { 5. Módulo de } \\
\text { elasticidade à } \\
\text { flexão estática }\end{array}$ & $\mathrm{E}_{\mathrm{M} 0}$ & $\begin{array}{l}\text { É a rigidez da madeira à flexão estática dada } \\
\text { pela carga aplicada no meio do vão livre } \\
\text { (distância entre apoios) do corpo de prova. } \\
\text { Para o cálculo, considera-se o quociente } \\
\text { entre o produto da carga máxima aplicada } \\
\text { pelo vão livre ao cubo e a flecha, ou } \\
\text { deslocamento no meio do vão livre, } \\
\text { provocado pela carga máxima aplicada na } \\
\text { seção transversal do corpo de prova. }\end{array}$ & $\mathrm{E}_{\mathrm{M} 0}=\frac{\mathrm{F}_{\max } \mathrm{L}^{3}}{4 \mathrm{fbh}^{3}}$ & $\begin{array}{l}\mathrm{E}_{\mathrm{M} 0}=\text { módulo de elasticidade à flexão } \\
\text { estática, em N.m }{ }^{-2} \text {, ou MPa, ao t.u. de } 12 \% \text {. } \\
F_{\text {max }}=\text { carga máxima aplicada, em N. } \\
L=\text { vão livre, em m, do corpo de prova. } \\
f=\text { flecha, em m, no meio do vão livre do } \\
\text { corpo de prova. } \\
b \text { e } h=\text { lados da seção transversal, em m, no } \\
\text { meio do vão livre do corpo de prova. }\end{array}$ \\
\hline $\begin{array}{l}\text { 6. Resistência à } \\
\text { compressão } \\
\text { paralela às } \\
\text { fibras }\end{array}$ & $\mathrm{f}_{\mathrm{c} 0}$ & $\begin{array}{l}\text { E a resistência à ruptura da madeira à } \\
\text { compressão paralela às fibras dada pelo } \\
\text { quociente entre a máxima força de } \\
\text { compressão que pode atuar na direção } \\
\text { paralela às fibras de um corpo de prova e } \\
\text { a área da sua seção transversal. }\end{array}$ & $\mathrm{f}_{\mathrm{c} 0}=\frac{\mathrm{C}_{\max }}{\mathrm{A}}$ & $\begin{array}{l}\mathrm{f}_{\mathrm{c} 0}=\text { resistência à compressão paralela às } \\
\text { fibras, em N.m }{ }^{-2} \text {, ou } \mathrm{MPa} \text {, ao t.u. de } 12 \% \text {. } \\
C_{\max }=\text { máxima força de compressão, em } \mathrm{N} \text {. } \\
A=\text { área da seção transversal, em } \mathrm{m}^{2} \text {, do } \\
\text { corpo de prova. }\end{array}$ \\
\hline $\begin{array}{l}\text { 7. Resistência } \\
\text { no limite } \\
\text { proporcional à } \\
\text { compressão } \\
\text { perpendicular às } \\
\text { fibras }\end{array}$ & $\mathrm{E}_{\mathrm{c} 90}$ & $\begin{array}{l}\text { É a resistência da madeira no limite } \\
\text { proporcional à compressão perpendicular } \\
\text { às fibras dada pelo quociente entre a força } \\
\text { no limite da proporcionalidade que pode } \\
\text { atuar na direção perpendicular às fibras da } \\
\text { madeira de um corpo de prova e a área da } \\
\text { seção de aplicação da carga. }\end{array}$ & $\mathrm{E}_{\mathrm{c} 90}=\frac{\mathrm{C}}{\mathrm{A}}$ & $\begin{array}{l}\mathrm{E}_{\mathrm{c} 90}=\text { resistência no limite proporcional à } \\
\text { compressão perpendicular às fibras, em N.m } \\
2 \text {, ou } \mathrm{MPa} \text {, ao t.u. de } 12 \% \text {. } \\
C=\text { força de compressão, em N. } \\
A=\text { área da seção de aplicação da carga, em } \\
\mathrm{m}^{2} \text {, do corpo de prova. }\end{array}$ \\
\hline $\begin{array}{l}\text { 8. Dureza Janka } \\
\text { paralela às } \\
\text { fibras }\end{array}$ & $\mathrm{f}_{\mathrm{H} 0}$ & $\begin{array}{l}\text { A dureza Janka paralela às fibras da } \\
\text { madeira é determinada convencionalmente } \\
\text { pela máxima força de compressão que atua } \\
\text { na face paralela às fibras de um corpo de } \\
\text { prova prismático e que produz a penetração } \\
\text { da área da seção diametral de uma semi- } \\
\text { esfera de aço de um centímetro quadrado na } \\
\text { profundidade igual a seu raio. }\end{array}$ & $\mathrm{f}_{\mathrm{H} 0}=F \mathrm{p}_{\max }$ & $\begin{array}{l}\mathrm{f}_{\mathrm{H} 0}=\text { dureza Janka paralela às fibras da } \\
\text { madeira, em N, ao t.u. de } 12 \% \text {. } \\
F p_{\max }=\text { máxima força de compressão atuante } \\
\text { na face paralela às fibras, em N. }\end{array}$ \\
\hline $\begin{array}{l}\text { 9. Dureza Janka } \\
\text { transversal às } \\
\text { fibras }\end{array}$ & $\mathrm{f}_{\mathrm{H} 90}$ & $\begin{array}{l}\text { A dureza Janka transversal às fibras da } \\
\text { madeira é determinada convencionalmente } \\
\text { pela máxima força de compressão que atua } \\
\text { na face transversal às fibras de um corpo de } \\
\text { prova prismático e que produz a penetração } \\
\text { da área da seção diametral de uma semi- } \\
\text { esfera de aço de um centímetro quadrado na } \\
\text { profundidade igual a seu raio. }\end{array}$ & $\mathrm{f}_{\mathrm{H} 90}=\mathrm{Ft}_{\max }$ & $\begin{array}{l}\mathrm{f}_{\mathrm{H} 90}=\text { dureza Janka da madeira transversal às } \\
\text { fibras, em N, ao t.u. de } 12 \% \\
F t_{\max }=\text { máxima força de compressão atuante } \\
\text { na face transversal às fibras, em N }\end{array}$ \\
\hline $\begin{array}{l}\text { 10. Resistência } \\
\text { à tração } \\
\text { perpendicular às } \\
\text { fibras }\end{array}$ & $f_{\mathrm{w} t 90}$ & $\begin{array}{l}\text { E a resistência à ruptura da madeira à } \\
\text { tração perpendicular às fibras e dada pela } \\
\text { máxima força de tração que pode atuar } \\
\text { em um corpo de prova alongado com } \\
\text { trecho central com área da seção } \\
\text { transversal uniforme. }\end{array}$ & $\mathrm{f}_{\mathrm{w} t 90}=\frac{\mathrm{Ftr}_{\max }}{\mathrm{A}}$ & $\begin{array}{l}\mathrm{f}_{\text {wt } 90}=\text { resistência à tração perpendicular às } \\
\text { fibras, em N.m }{ }^{-2} \text {, ou } \mathrm{MPa} \text {, ao t.u. de } 12 \% . \\
F t r_{\max }=\text { máxima força de tração, em } \mathrm{N} . \\
A=\text { área da seção transversal uniforme, em } \\
\mathrm{m}^{2} \text {, do corpo de prova. }\end{array}$ \\
\hline
\end{tabular}




\begin{tabular}{|c|c|c|c|c|}
\hline Propriedade & Notação & Descrição sumária & Fórmula de cálculo & Componentes da fórmula \\
\hline $\begin{array}{l}\text { 11. Resistência } \\
\text { ao } \\
\text { fendilhamento }\end{array}$ & $\mathrm{f}_{\mathrm{v} 0}$ & $\begin{array}{l}\text { É a resistência à ruptura da madeira ao } \\
\text { fendilhamento e dada pela máxima força } \\
\text { de fendilhamento que pode atuar na área } \\
\text { da seção transversal crítica de um corpo } \\
\text { de prova. }\end{array}$ & $\mathrm{f}_{\mathrm{v} 0}=\frac{\mathrm{Ff}}{\mathrm{A}}$ & $\begin{array}{l}\mathrm{f}_{\mathrm{v} 0}=\text { resistência ao fendilhamento, em N.m } \\
\text { ou } \mathrm{MPa} \text {, ao t.u. de } 12 \% \text {. } \\
F f_{\max }=\text { máxima força de fendilhamento, em } \\
\mathrm{N} . \\
A=\text { área da seção transversal crítica, } \mathrm{em} \mathrm{m}^{2}, \\
\text { do corpo de prova. }\end{array}$ \\
\hline $\begin{array}{l}\text { 12. Resistência } \\
\text { ao cisalhamento }\end{array}$ & $f_{s 0}$ & $\begin{array}{l}\text { É a resistência à ruptura da madeira ao } \\
\text { cisalhamento e dada pela máxima força } \\
\text { de cisalhamento que pode atuar na área } \\
\text { da seção transversal crítica de um corpo } \\
\text { de prova. }\end{array}$ & $\mathrm{f}_{\mathrm{s} 0}=\frac{\mathrm{Fc}_{\max }}{\mathrm{A}}$ & $\begin{array}{l}\mathrm{f}_{\mathrm{s} 0}=\text { resistência ao cisalhamento, em N. } \mathrm{m}^{-2} \text {, } \\
\text { ou } \mathrm{MPa} \text {, ao t.u. de } 12 \% \text {. } \\
F c_{\max }=\text { máxima força de cisalhamento, em } \mathrm{N} \text {. } \\
A=\text { área da seção transversal crítica, em m }{ }^{2} \text {, } \\
\text { do corpo de prova. }\end{array}$ \\
\hline
\end{tabular}

t.u.: teor de umidade; N: Newton; MPa: MegaPascal.

\section{Amostragem}

Os dados básicos foram obtidos a partir de uma amostra procedente de quatro publicações do Laboratório de Produtos Florestais/Instituto Brasileiro do Meio Ambiente e dos Recursos Naturais Renováveis (LPF/IBAMA) (IBAMA, 1997; INSTITUTO, 1981; INSTITUTO, 1988; SOUZA et al., 1997). Tais dados foram determinados utilizando-se as normas da COPANT, as quais são praticamente iguais às da ASTM (INSTITUTO, 1988), sendo que as propriedades mecânicas foram obtidas com corpos de prova com um teor de umidade de $12 \%$.

A amostra totalizou informações de 163 espécies tropicais brasileiras (Tabela 3), possuindo boa representação da variabilidade entre as madeiras (por exemplo, os dados de $\rho_{\text {bas }}$ variam de 0,29 a 1,01 g. $\mathrm{cm}^{-3}$ ). Ainda que existam informações disponíveis em diferentes fontes bibliográficas acerca das PFMM de outras espécies brasileiras, o que possibilitaria expandir a amostra de dados, optou-se por obtê-las de apenas uma fonte, o LPF/IBAMA, por se considerar que, desse modo, os dados são dotados de maior homogeneidade, uma vez que foram obtidos pelos mesmos métodos de ensaio e, presumivelmente, pelas mesmas máquinas.

Visando uniformização aos padrões internacionais para PFMM, a unidade de medida das propriedades $\mathrm{f}_{\mathrm{M}}, \mathrm{f}_{\mathrm{c} 0}, \mathrm{E}_{\mathrm{c} 90}, \mathrm{f}_{\mathrm{w} t 90}, \mathrm{f}_{\mathrm{v} 0}$ e $\mathrm{f}_{\mathrm{s} 0}$, que é apresentada nas fontes bibliográficas em quilograma-força por centímetro quadrado $\left(\mathrm{kgf}_{\mathrm{cm}} \mathrm{cm}^{-2}\right)$, foi convertida para MegaPascal (MPa), utilizando-se o fator 0,0980665. A unidade de medida de $\mathrm{E}_{\mathrm{M} 0}$, que na origem é expressa em $1000 \mathrm{kgf.cm}{ }^{-2}$, foi convertida para MPa pelo fator 98,0665. A unidade de medida de $\mathrm{f}_{\mathrm{H} 0}$ e $\mathrm{f}_{\mathrm{H} 90}$, que é apresentada em kgf, foi convertida para Newton (N) pelo fator 9,80665 .

Tabela 3. Propriedades físicas e mecânicas de 163 espécies de madeiras tropicais brasileiras.

Table 3. Physical and mechanical properties of the 163 species of Brazilian tropical wood.

\begin{tabular}{|c|c|c|c|c|c|c|c|c|c|c|c|c|c|c|}
\hline $\mathbf{n}$ & $\begin{array}{l}\text { Nome } \\
\text { usual }\end{array}$ & Nome científico & $\rho_{\text {bas }}$ & $\varepsilon_{\mathbf{r}, 3}$ & $\begin{array}{r}\varepsilon_{\mathrm{r}} \\
2\end{array}$ & $\mathbf{f}_{\mathbf{M}}$ & $\mathbf{E}_{\mathbf{M 0}}$ & $\mathbf{f}_{\mathrm{c} 0}$ & $\mathbf{E}_{\mathrm{c90}}$ & $\mathbf{f}_{\mathrm{H} 0}$ & $\mathbf{f}_{\mathrm{H} 90}$ & $\begin{array}{r}\mathbf{f}_{\mathrm{wt}} \\
90 \\
\end{array}$ & $\mathbf{f}_{\mathrm{v} 0}$ & $\mathbf{f}_{\mathrm{s} 0}$ \\
\hline 1 & Abiu-branco & Syzygiopsis oppositifolia Ducke & 0,65 & 7,8 & 4,6 & - & - & - & - & - & - & - & - & - \\
\hline 2 & $\begin{array}{l}\text { Abiu-casca- } \\
\text { grossa }\end{array}$ & $\begin{array}{l}\text { Planchonella pachycarpa Pires } \\
\text { (ined.) }\end{array}$ & 0,74 & 12,6 & 6,5 & 161,61 & 16867 & 82,57 & 10,98 & 12092 & 10719 & 4,61 & 8,04 & 16,77 \\
\hline 3 & $\begin{array}{l}\text { Abiu- } \\
\text { pitomba }\end{array}$ & $\begin{array}{l}\text { Sandwithiodoxa egregia } \\
\text { (Sandw.)Aubr. \& Pellegr. }\end{array}$ & 0,84 & 11,2 & 6,8 & 171,81 & 17554 & 88,95 & 14,71 & 14524 & 13121 & 5,69 & 8,83 & 19,02 \\
\hline 4 & $\begin{array}{l}\text { Abiurana- } \\
\text { branca }\end{array}$ & $\begin{array}{l}\text { Franchetella gongrijpii (Eyma) } \\
\text { Aubrév. }\end{array}$ & 0,72 & 10,1 & 4,5 & 144,45 & 15298 & 75,51 & 12,55 & 11670 & 10660 & 4,81 & 7,85 & 17,65 \\
\hline 5 & $\begin{array}{l}\text { Abiurana- } \\
\text { seca }\end{array}$ & Diploon venezuelana Aubrév. & 0,85 & 10,1 & 7,1 & 186,91 & 17750 & 96,40 & 19,42 & 11827 & 13102 & 3,14 & 5,39 & 19,61 \\
\hline 6 & $\begin{array}{l}\text { Abiurana- } \\
\text { vermelha }\end{array}$ & Pouteria caimito (R. \& P.) Radlk. & 0,88 & 9,4 & 5,3 & 168,48 & 17554 & 87,67 & 16,28 & 12798 & 12239 & 4,90 & 5,69 & 16,38 \\
\hline 7 & Açacu & Hura crepitans $\mathrm{L}$. & 0,39 & 5,2 & 3,7 & 67,67 & 8434 & 32,95 & 4,71 & 3844 & 2775 & 2,55 & 3,33 & 6,96 \\
\hline 8 & Achichá & Sterculia speciosa K. Schum. & 0,49 & 10,6 & 5,2 & 90,52 & 13631 & 46,48 & 4,81 & 4992 & 4158 & 2,35 & 4,22 & 8,73 \\
\hline 9 & $\begin{array}{l}\text { Açoita- } \\
\text { cavalo }\end{array}$ & Lueheopsis duckeana Burret & 0,62 & 9,5 & 4,6 & 123,17 & 14220 & 62,47 & 9,22 & 8865 & 7708 & 4,31 & 4,61 & 12,55 \\
\hline 10 & $\begin{array}{l}\text { Amapá- } \\
\text { amargoso }\end{array}$ & $\begin{array}{l}\text { Parahancornia amapa (Huber) } \\
\text { Ducke }\end{array}$ & 0,46 & 6,8 & 3,7 & 89,04 & 11082 & 45,90 & 5,98 & 5296 & 3511 & 3,92 & 5,10 & 9,61 \\
\hline 11 & Amapá-doce & Brosimum potabile Ducke & 0,53 & - & - & 97,18 & 10787 & 55,02 & 8,24 & 7247 & 5266 & 3,14 & - & 10,20 \\
\hline 12 & $\begin{array}{l}\text { Amapá- } \\
\text { doce/mururé- } \\
\text { rana }\end{array}$ & Brosimum parinarioides Ducke & 0,57 & - & - & 102,28 & 11278 & 56,98 & 8,04 & 7198 & 5560 & 2,94 & - & 10,00 \\
\hline 13 & Amoreira & $\begin{array}{l}\text { Maclura tinctoria }(\text { L.) D. Don ex } \\
\text { Steud. }\end{array}$ & 0,73 & 5,9 & 3,2 & 153,47 & 12651 & 86,10 & 22,36 & 11415 & 10611 & 5,30 & 6,86 & 15,59 \\
\hline 14 & Anani & Symphonia globulifera $\mathrm{L}$. & 0,58 & 7,9 & 4,5 & 109,25 & 13729 & 61,29 & 7,16 & 6669 & 5600 & 3,24 & 3,24 & 10,40 \\
\hline 15 & Andiroba & Carapa guianensis Aubl. & 0,59 & 8,1 & 4,4 & 107,19 & 11768 & 59,72 & 8,83 & 8247 & 6276 & 4,02 & - & 10,89 \\
\hline
\end{tabular}




\begin{tabular}{|c|c|c|c|c|c|c|c|c|c|c|c|c|c|c|}
\hline n & $\begin{array}{l}\text { Nome } \\
\text { usual }\end{array}$ & Nome científico & $\rho_{\text {bas }}$ & $\varepsilon_{\mathrm{r}, 3}$ & $\begin{array}{r}\varepsilon_{\mathrm{r}}, \\
2\end{array}$ & $\mathbf{f}_{\mathbf{M}}$ & $\mathbf{E}_{\mathbf{M 0}}$ & $\mathbf{f}_{\mathrm{c} 0}$ & $\mathbf{E}_{\mathbf{c 9 0}}$ & $\mathbf{f}_{\mathrm{H} 0}$ & $\mathbf{f}_{\mathrm{H} 90}$ & $\begin{array}{r}\mathbf{f}_{\mathrm{wt}} \\
90 \\
\end{array}$ & $\mathbf{f}_{\mathrm{v} 0}$ & $\mathbf{f}_{\mathrm{s} 0}$ \\
\hline 16 & $\begin{array}{l}\text { Angelim-da- } \\
\text { mata }\end{array}$ & Hymenolobium sp. & 0,60 & 7,2 & 3,7 & 111,89 & 11866 & 56,29 & 11,18 & 8140 & 6325 & 4,71 & 5,49 & 13,04 \\
\hline 17 & $\begin{array}{l}\text { Angelim-da- } \\
\text { mata }\end{array}$ & Hymenolobium modestum Ducke & 0,65 & 7,7 & 4,6 & 118,46 & 13239 & 59,92 & 10,49 & 7904 & 7326 & 3,82 & 5,39 & 13,73 \\
\hline 18 & $\begin{array}{l}\text { Angelim- } \\
\text { pedra }\end{array}$ & Hymenolobium petraeum Ducke & 0,59 & 6,3 & 4,1 & 109,34 & 11572 & 52,27 & 11,28 & 7659 & 5786 & 3,82 & - & 12,26 \\
\hline 19 & $\begin{array}{l}\text { Angelim- } \\
\text { pedra }\end{array}$ & Dinizia excelsa Ducke & 0,83 & 9,5 & 5,7 & 156,91 & 16966 & 85,61 & 14,81 & 14318 & 13543 & 3,82 & 6,57 & 17,65 \\
\hline 20 & $\begin{array}{l}\text { Angelim- } \\
\text { rajado }\end{array}$ & $\begin{array}{l}\text { Marmaroxylon racemosum (Ducke) } \\
\text { Killip. ex Record. }\end{array}$ & 0,79 & 9,3 & 5,9 & 163,57 & 16377 & 79,14 & 18,93 & 14886 & 14131 & 2,84 & - & 17,26 \\
\hline 21 & Araracanga & $\begin{array}{l}\text { Aspidosperma desmanthum Benth. } \\
\text { ex Müell. Arg. }\end{array}$ & 0,69 & 9,0 & 5,8 & 132,98 & 14612 & 67,86 & 11,87 & 9248 & 7816 & 2,94 & 5,49 & 12,65 \\
\hline 22 & Axixá & Sterculia apeibophylla Ducke & 0,47 & 10,6 & 5,4 & 84,24 & 10493 & 46,09 & 4,90 & 4080 & 3050 & 3,14 & 4,61 & 8,83 \\
\hline 23 & Bacuri & Platonia insignis Mart. & 0,67 & 8,1 & 4,6 & - & - & - & - & - & - & - & - & - \\
\hline 24 & Barrote & $\begin{array}{l}\text { Tetragastris panamensis (Engl.) O. } \\
\text { Kuntze }\end{array}$ & 0,75 & 9,8 & 5,0 & 123,56 & 14024 & 60,51 & 12,85 & 13876 & 12670 & 5,00 & 6,18 & 18,93 \\
\hline 25 & $\begin{array}{l}\text { Breu/Amescl } \\
\text { a }\end{array}$ & Trattinickia cf. burseraefolia Mart. & 0,50 & 6,8 & 4,1 & 64,14 & 10983 & 45,11 & 5,79 & 5099 & - & 3,63 & 5,69 & 9,90 \\
\hline 26 & $\begin{array}{l}\text { Breu/breu- } \\
\text { branco- } \\
\text { campo }\end{array}$ & $\begin{array}{l}\text { Protium heptaphyllum (Aubl.) } \\
\text { March var brasiliense Engl. }\end{array}$ & 0,55 & - & - & 85,12 & 9807 & 48,05 & 6,77 & 4452 & 3903 & 5,39 & - & 9,81 \\
\hline 27 & Breu-manga & $\begin{array}{l}\text { Tetragastris altissima (Aubl.) } \\
\text { Swartz }\end{array}$ & 0,74 & 9,0 & 4,6 & 134,65 & 14318 & 76,39 & 15,20 & 12013 & 9650 & 6,47 & 6,86 & 17,95 \\
\hline 28 & $\begin{array}{l}\text { Breu- } \\
\text { manga/breu }\end{array}$ & Protium sp. & 0,76 & 9,8 & 5,1 & 137,98 & 15495 & 78,94 & 11,57 & 12239 & 10307 & 4,71 & 6,57 & 17,85 \\
\hline 29 & Breu-preto & $\begin{array}{l}\text { Tetragastris panamensis (Engl.) O. } \\
\text { Kuntze }\end{array}$ & 0,77 & 9,9 & 5,1 & 122,49 & 13239 & 68,74 & 14,12 & 13582 & 11287 & 5,30 & 7,94 & 20,10 \\
\hline 30 & $\begin{array}{l}\text { Breu- } \\
\text { preto/breu }\end{array}$ & Protium tenuifolium (Engl.) Engl. & 0,63 & 8,4 & 4,2 & 114,15 & 12651 & 63,25 & 9,22 & 8983 & 6747 & 4,90 & 5,98 & 14,61 \\
\hline 31 & Burra-leiteira & Sapium marmieri Huber & 0,39 & 7,5 & 5,6 & 62,66 & 8924 & 32,56 & 3,73 & 2295 & 1442 & 2,75 & 3,73 & 6,57 \\
\hline 32 & $\begin{array}{l}\text { Cabreuva- } \\
\text { parda }\end{array}$ & Myrocarpus frondosus Allemão & 0,78 & 7,0 & 4,4 & 154,16 & 15102 & 85,91 & 13,53 & 15289 & 13680 & 4,02 & 5,69 & 17,85 \\
\hline 33 & Caja & Spondias lutea $\mathrm{L}$. & 0,38 & 7,4 & 4,2 & 46,78 & 7747 & 30,89 & 3,73 & 2108 & 1196 & 2,84 & 3,43 & 5,79 \\
\hline 34 & Cajuaçu & Anacardium giganteum Hancock. & - & 6,3 & 3,4 & - & - & - & - & - & - & - & - & - \\
\hline 35 & Cajuaçu & Anacardium spruceanum Benth. & 0,42 & 6,0 & 2,9 & 64,14 & 9807 & 36,48 & 4,41 & 3825 & 2491 & 2,84 & - & 6,77 \\
\hline 36 & Canafistula & Cassia fastuosa Willd. & 0,71 & 9,1 & 6,2 & 113,95 & 13141 & 72,18 & 12,75 & 12258 & 11121 & 3,24 & 4,61 & 14,42 \\
\hline 37 & Caraipé & $\begin{array}{l}\text { Licania octandra (Hoffm. ex Roem } \\
\text { \& Schult) O. Kuntze }\end{array}$ & 0,77 & 11,9 & 6,1 & 161,03 & 17064 & 83,36 & 11,47 & 12199 & 12249 & 4,02 & 4,81 & 15,20 \\
\hline 38 & $\begin{array}{l}\text { Castanha- } \\
\text { arara/Mungu } \\
\text { ba }\end{array}$ & Joannesia heveoides Ducke & 0,39 & - & - & 63,65 & 8238 & 34,42 & 4,81 & 3844 & 2589 & 3,24 & - & 5,88 \\
\hline 39 & $\begin{array}{l}\text { Castanha-de- } \\
\text { cutia }\end{array}$ & Couepia robusta Huber & 0,83 & 11,0 & 5,8 & 154,55 & 15593 & 78,85 & 19,02 & 13347 & 14259 & 3,92 & 5,79 & 13,04 \\
\hline 40 & $\begin{array}{l}\text { Castanha- } \\
\text { sapucaia }\end{array}$ & Lecythis pisonis Cambess. & 0,84 & 8,0 & 5,6 & 149,94 & 14808 & 70,22 & 14,91 & 12994 & 12592 & 4,22 & 5,79 & 17,16 \\
\hline 41 & Castanheira & $\begin{array}{l}\text { Bertholletia excelsa Humb. \& } \\
\text { Bonpl. }\end{array}$ & 0,63 & - & - & 116,01 & 12553 & 58,35 & 9,90 & 8071 & 6541 & 4,22 & - & 11,47 \\
\hline 42 & Caucho & Castilla ulei Warb. & 0,39 & 10,5 & 6,2 & 55,80 & 8924 & 36,77 & 3,63 & 2716 & 1393 & 1,86 & 2,94 & 5,88 \\
\hline 43 & Cedrorana & Cedrelinga catenaeformis Ducke & 0,44 & 7,9 & 4,8 & 77,77 & 12847 & 46,58 & 3,63 & 3962 & 3785 & 4,51 & - & 7,16 \\
\hline 44 & Conduru & Brosimum rubescens Taub. & 0,71 & 8,1 & 5,3 & 147,49 & 16377 & 76,59 & 12,36 & 10650 & 9346 & 3,92 & 5,69 & 12,55 \\
\hline 45 & Copaiba & $\begin{array}{l}\text { Copaifera duckei Dwyer/C. } \\
\text { reticulata Ducke }\end{array}$ & 0,62 & 8,2 & 4,1 & 115,62 & 12062 & 58,84 & 9,71 & 8502 & 6512 & 4,31 & - & 11,96 \\
\hline 46 & Copaiba & Copaifera langsdorfii Desf. & - & 6,7 & 4,1 & - & - & - & - & - & - & - & - & - \\
\hline 47 & Cuia & $\begin{array}{l}\text { Terminalia cf. argentea Mart. \& } \\
\text { Zucc. }\end{array}$ & 0,80 & 9,8 & 5,8 & 142,00 & 15396 & 84,63 & 12,85 & 15416 & 13965 & 4,41 & 5,88 & 17,65 \\
\hline 48 & Cuiarana & $\begin{array}{l}\text { Terminalia amazonica (Gmell) } \\
\text { Exell. }\end{array}$ & 0,80 & - & - & 146,02 & 14024 & 77,96 & 14,02 & 11435 & 9944 & 5,20 & - & 13,93 \\
\hline 49 & Cumaru & Dipteryx odorata Willd. & 0,91 & 8,4 & 5,4 & 172,99 & 17946 & 96,79 & 20,59 & 13131 & 15700 & 4,81 & 8,53 & 21,97 \\
\hline 50 & Cupiúba & Goupia glabra Aubl. & 0,71 & 8,4 & 4,5 & 131,41 & 14612 & 67,57 & 14,51 & 9993 & 8140 & 4,12 & 5,49 & 14,51 \\
\hline 51 & Envira & Rollinia exsucca (Dun.) A. DC. & 0,52 & 8,8 & 3,2 & 89,63 & 11768 & 52,27 & - & 5286 & 3844 & - & 2,94 & 8,43 \\
\hline 52 & $\begin{array}{l}\text { Envira- } \\
\text { branca }\end{array}$ & Xylopia nitida Dun. & 0,56 & 11,4 & 6,7 & 116,99 & 15887 & 62,08 & 5,69 & 6561 & 4972 & 2,26 & 4,71 & 8,43 \\
\hline 53 & Envira-preta & Guatteria olivacea R. E. Fries & 0,51 & 9,3 & 4,4 & 98,26 & 12258 & 51,39 & - & 5806 & 4756 & 3,82 & - & 8,73 \\
\hline 54 & Envira-preta & Guatteria procera R. E. Fries & 0,65 & 11,4 & 7,2 & 121,50 & 15298 & 69,14 & 5,39 & 7443 & 6531 & 2,65 & 4,90 & 10,49 \\
\hline 55 & Envira-preta & $\begin{array}{l}\text { Onychopetalum amazonicum R. E. } \\
\text { Fries }\end{array}$ & 0,57 & 8,1 & 4,9 & 122,78 & 15004 & 65,21 & 6,47 & 6669 & 6600 & 2,35 & 5,00 & 10,20 \\
\hline 56 & Envira-preta & $\begin{array}{l}\text { Diclinanona calycina (Diels) R.E. } \\
\text { Fries }\end{array}$ & 0,47 & - & - & 93,56 & 11082 & 48,84 & - & 5060 & 3952 & 3,04 & - & 7,45 \\
\hline 57 & $\begin{array}{l}\text { Espinheiro- } \\
\text { preto }\end{array}$ & Acacia polyphylla A.DC. & 0,60 & 10,1 & 4,9 & 104,54 & 12945 & 57,86 & 8,53 & 6776 & 4864 & 3,43 & 6,37 & 11,47 \\
\hline 58 & $\begin{array}{l}\text { Faeira/louro- } \\
\text { faia }\end{array}$ & Roupala montana Aubl. & 0,77 & 12,3 & 6,3 & 158,28 & 16966 & 82,96 & 10,98 & 9571 & 9650 & 6,18 & 8,53 & 15,40 \\
\hline 59 & $\begin{array}{l}\text { Fava- } \\
\text { amargosa }\end{array}$ & Vatairea paraensis Ducke & 0,78 & 9,8 & 4,8 & 148,37 & 15004 & 77,77 & 12,85 & 9159 & 9669 & 4,12 & 6,37 & 15,79 \\
\hline 60 & $\begin{array}{l}\text { Fava-arara- } \\
\text { tucupi/faveira }\end{array}$ & Parkia paraensis Ducke & 0,44 & 7,6 & 3,5 & 73,55 & 11474 & 38,64 & 4,61 & 3913 & 3305 & 3,43 & 5,59 & 9,61 \\
\hline
\end{tabular}




\begin{tabular}{|c|c|c|c|c|c|c|c|c|c|c|c|c|c|c|}
\hline $\mathbf{n}$ & $\begin{array}{l}\text { Nome } \\
\text { usual }\end{array}$ & Nome científico & $\rho_{\text {bas }}$ & $\varepsilon_{\mathrm{r}, 3}$ & $\begin{array}{r}\varepsilon_{\mathrm{r}} \\
2\end{array}$ & $\mathbf{f}_{\mathbf{M}}$ & $\mathbf{E}_{\mathbf{M} 0}$ & $\mathbf{f}_{\mathrm{c} 0}$ & $\mathbf{E}_{\mathbf{c 9 0}}$ & $\mathbf{f}_{\mathbf{H} 0}$ & $\mathbf{f}_{\mathrm{H} 90}$ & $\begin{array}{r}f_{w t} \\
90 \\
\end{array}$ & $\mathbf{f}_{\mathrm{v} 0}$ & $\mathbf{f}_{\mathrm{s} 0}$ \\
\hline 61 & Fava-branca & $\begin{array}{l}\text { Stryphnodendron pulcherrimum } \\
\text { (Willd.) Hochr. }\end{array}$ & 0,48 & 7,5 & 5,1 & 95,91 & 11866 & 44,91 & 4,81 & 7306 & 5825 & 3,04 & 3,63 & 12,26 \\
\hline 62 & $\begin{array}{l}\text { Fava-de- } \\
\text { rosca }\end{array}$ & Enterolobium schomburgkii Benth. & 0,84 & 9,3 & 4,2 & 161,61 & 16769 & 78,65 & 14,81 & 9277 & 10434 & 5,49 & 7,94 & 17,36 \\
\hline 63 & $\begin{array}{l}\text { Fava-folha- } \\
\text { fina/timbora } \\
\text { na }\end{array}$ & Piptadenia suaveolens Miq. & 0,76 & 8,3 & 5,1 & 146,90 & 15396 & 78,26 & 13,93 & 8806 & 9601 & 5,30 & 6,67 & 15,89 \\
\hline 64 & $\begin{array}{l}\text { Fava- } \\
\text { tamboril/tam } \\
\text { boril }\end{array}$ & Enterolobium maximum Ducke & 0,37 & 4,5 & 2,4 & 48,84 & 7649 & 37,66 & 4,12 & 3854 & 2824 & 2,26 & 3,14 & 8,83 \\
\hline 65 & Faveira & $\begin{array}{l}\text { Parkia oppositifolia Spruce ex. } \\
\text { Benth. }\end{array}$ & 0,42 & 5,4 & 2,7 & 81,98 & 9512 & 34,81 & 5,49 & 4050 & 2854 & 3,14 & 3,82 & 9,41 \\
\hline 66 & $\begin{array}{l}\text { Faveira } \\
\text { bolacha }\end{array}$ & Vatairea sericea Ducke & 0,71 & 8,4 & 4,5 & 135,43 & 14906 & 64,82 & 11,18 & 7649 & 7894 & 3,53 & 4,90 & 13,83 \\
\hline 67 & $\begin{array}{l}\text { Faveira- } \\
\text { bolota/faveir } \\
\text { a }\end{array}$ & Parkia pendula Benth. ex Walp. & 0,50 & 7,8 & 3,8 & 84,73 & 11572 & 48,25 & 7,06 & 5345 & 4207 & 3,92 & 5,00 & 11,28 \\
\hline 68 & $\begin{array}{l}\text { Faveira- } \\
\text { branca }\end{array}$ & Parkia multijuga Benth. & 0,38 & 7,0 & 2,9 & 60,61 & 8630 & 36,97 & 5,59 & 4011 & 2971 & 3,82 & - & 8,04 \\
\hline 69 & $\begin{array}{l}\text { Faveira- } \\
\text { folha-fina }\end{array}$ & Piptadenia communis Benth. & 0,68 & - & - & 116,01 & 13435 & 68,65 & 11,47 & 7218 & 7424 & 3,73 & - & 13,14 \\
\hline 70 & Figueira & Ficus insipida Willd. & 0,36 & 9,7 & 5,0 & 61,59 & 7943 & 37,66 & 3,92 & 2079 & 1196 & 1,96 & 3,04 & 6,47 \\
\hline 71 & Freijó & Cordia bicolor D.C. & 0,49 & - & - & 81,30 & 11866 & 49,92 & 3,73 & 5619 & 4286 & 3,33 & - & 7,65 \\
\hline 72 & Freijó & Cordia sagotii I.M. Johnston & 0,50 & - & - & 86,40 & 9807 & 47,86 & - & 5148 & 3923 & 3,43 & - & 7,55 \\
\hline 73 & $\begin{array}{l}\text { Freijó/frei } \\
\text { jorge }\end{array}$ & Cordia goeldiana Huber & 0,48 & - & - & 91,40 & 10199 & 50,70 & 6,08 & 5962 & 4433 & 3,04 & - & 8,34 \\
\hline 74 & Garapeira & Apuleia molaris Spruce ex. Benth. & 0,75 & 10,1 & 6,5 & 124,74 & 12651 & 63,15 & 15,49 & 7306 & 8287 & 5,49 & 6,67 & 12,85 \\
\hline 75 & Glícia & Glycydendron amazonicum Ducke & 0,66 & 7,6 & 4,9 & 125,92 & 14906 & 65,02 & 10,20 & 8561 & 7433 & 3,33 & 4,51 & 13,44 \\
\hline 76 & Goiabão & Pouteria pachycarpa Pires & 0,73 & 11,2 & 6,2 & 155,53 & 16377 & 74,04 & 11,57 & 15220 & 12817 & 5,79 & 6,28 & 18,14 \\
\hline 77 & Guariúba & Clarisia racemosa Ruiz \& Pav. & 0,60 & 6,2 & 3,1 & 108,85 & 12160 & 64,53 & 9,32 & 7836 & 6119 & 2,84 & 5,00 & 11,67 \\
\hline 78 & Ingá & Inga alba Willd. & 0,62 & 9,6 & 4,0 & 120,72 & 13337 & 60,21 & 8,14 & 8463 & 6306 & 4,51 & 7,06 & 14,32 \\
\hline 79 & $\begin{array}{l}\text { Ingá-de- } \\
\text { porco }\end{array}$ & $\begin{array}{l}\text { Macrosamanea pedicellaris (DC.) } \\
\text { Kleinh. }\end{array}$ & 0,49 & 7,6 & 4,3 & 97,28 & 11670 & 50,01 & 7,85 & 4746 & 4246 & 4,12 & 4,90 & 11,96 \\
\hline 80 & Ingarana & Inga $s p$. & 0,58 & 9,6 & 4,1 & 157,99 & 15495 & 82,08 & 13,44 & 14867 & 12660 & 7,65 & 6,96 & 20,20 \\
\hline 81 & Ingarana/ingá & Inga paraensis Ducke & 0,82 & 10,7 & 5,3 & 182,99 & 17946 & 83,36 & 14,42 & 14886 & 13631 & - & - & 21,38 \\
\hline 82 & Ipê & $\begin{array}{l}\text { Tabebuia serratifolia (Vahl.) } \\
\text { Nichols. }\end{array}$ & 0,87 & 10,1 & 7,4 & 200,64 & 16573 & 100,13 & 24,61 & 16328 & 14426 & - & - & 16,57 \\
\hline 83 & $\begin{array}{l}\text { Itaúba/itaúba } \\
\text {-abacate }\end{array}$ & $\begin{array}{l}\text { Mezilaurus lindaviana Schw. \& } \\
\text { Mez }\end{array}$ & 0,68 & - & - & 114,74 & 12553 & 56,19 & 9,51 & 4433 & 4413 & 4,02 & - & 8,43 \\
\hline 84 & $\begin{array}{l}\text { Itaúba- } \\
\text { amarela/itaúba }\end{array}$ & $\begin{array}{l}\text { Mezilaurus itauba (Meissn.) } \\
\text { Taubert ex Mez }\end{array}$ & 0,70 & - & - & 112,19 & 12062 & 57,17 & 10,79 & 5394 & 5796 & 4,61 & - & 10,10 \\
\hline 85 & Janitá & Brosimum alicastrum Swartz & 0,74 & 9,4 & 5,6 & 152,69 & 15985 & 86,20 & 11,18 & 11287 & 9159 & 4,41 & 6,86 & 14,81 \\
\hline 86 & Jará & Pouteria sp. & 0,72 & 9,7 & 5,9 & 155,24 & 16867 & 78,85 & 12,85 & 12013 & 10758 & 4,81 & 6,86 & 13,14 \\
\hline 87 & $\begin{array}{l}\text { Jutaí- } \\
\text { açu/jatobá }\end{array}$ & $\begin{array}{l}\text { Hymenaea courbaril L. var. } \\
\text { courbaril }\end{array}$ & 0,76 & 7,7 & 3,4 & 137,20 & 15593 & 75,81 & 13,83 & 12288 & 10944 & 6,67 & 7,45 & 19,02 \\
\hline 88 & $\begin{array}{l}\text { Jutaí- } \\
\text { mirim/jatobá }\end{array}$ & Hymenaea parvifolia Huber & 0,90 & 9,7 & 3,7 & 169,85 & 16966 & 94,73 & 18,83 & 16387 & 16867 & 6,47 & 7,16 & 23,14 \\
\hline 89 & $\begin{array}{l}\text { Jutaí- } \\
\text { pororoca }\end{array}$ & Dialium guianensis (Aubl.) Sandw. & 0,88 & 8,9 & 5,1 & 188,58 & 17162 & 90,42 & 21,87 & 16867 & 16122 & 4,41 & 7,16 & 22,36 \\
\hline 90 & Leiteiro & Sapium aereum Klotzch & 0,40 & 7,8 & 4,2 & 70,02 & 9905 & 43,44 & 5,10 & 2726 & 1687 & 2,75 & 3,63 & 6,86 \\
\hline 91 & $\begin{array}{l}\text { Louro- } \\
\text { amarelo/louro }\end{array}$ & Licaria rigida (Kosterm.) Kosterm. & 0,73 & 9,1 & 5,3 & 165,54 & 17554 & 85,51 & 12,75 & 10562 & 8983 & 5,30 & 5,59 & 16,77 \\
\hline 92 & $\begin{array}{l}\text { Louro- } \\
\text { canela/louro }\end{array}$ & Ocotea neesiana (Miq.) Kosterm. & 0,55 & 7,7 & 4,2 & 107,97 & 11866 & 60,02 & 7,16 & 4933 & 4491 & 5,49 & - & 12,45 \\
\hline 93 & $\begin{array}{l}\text { Louro- } \\
\text { canela/louro }\end{array}$ & Ocotea sp. & 0,63 & 7,8 & 3,6 & 119,74 & 14024 & 63,45 & 9,71 & 5237 & 5256 & 4,81 & 6,96 & 12,36 \\
\hline 94 & $\begin{array}{l}\text { Louro- } \\
\text { cunuaru }\end{array}$ & Ocotea sp. & 0,59 & 6,9 & 4,3 & 126,70 & 13729 & 61,98 & 12,06 & 5560 & 5403 & - & - & - \\
\hline 95 & $\begin{array}{l}\text { Louro- } \\
\text { vermelho }\end{array}$ & Nectandra rubra Mez. & 0,55 & 7,9 & 3,2 & 77,86 & 10689 & 49,92 & 4,81 & 3354 & 3364 & 2,94 & - & 7,35 \\
\hline 96 & $\begin{array}{l}\text { Maminha- } \\
\text { de-porca }\end{array}$ & Zanthoxylon regnelianum Engl. & 0,50 & 8,6 & 5,8 & 106,60 & 10787 & 51,48 & 7,55 & 5129 & 3521 & 3,33 & 5,49 & 9,61 \\
\hline 97 & Mandioqueira & Qualea cf. lancifolia Ducke & 0,58 & - & - & 107,58 & 12553 & 58,84 & 6,86 & 5747 & 4766 & 4,12 & - & 10,30 \\
\hline 98 & $\begin{array}{l}\text { Mandioqueir } \\
\mathrm{a} \text { áspera }\end{array}$ & Qualea brevipedicellata Stafleu & 0,74 & 8,6 & 4,4 & 132,00 & 15593 & 79,83 & 14,22 & 11032 & 9630 & 2,26 & 4,81 & 13,14 \\
\hline 99 & $\begin{array}{l}\text { Mandioqueir } \\
\text { a lisa }\end{array}$ & Qualea albiflora Warm. & 0,57 & 9,5 & 5,3 & 107,38 & 12847 & 57,27 & 7,75 & 8296 & 6011 & 4,41 & 5,20 & 13,04 \\
\hline 100 & $\begin{array}{l}\text { Mandioqueir } \\
\text { a-escamosa }\end{array}$ & Qualea dinizii Ducke & 0,54 & 9,1 & 4,6 & 102,38 & 11376 & 51,39 & 8,14 & - & 2658 & 3,82 & 5,30 & 9,81 \\
\hline 101 & Mangabarana & Pouteria anomala (Pires) Penning. & 0,73 & 9,1 & 5,7 & 161,71 & 15985 & 83,75 & 15,40 & 12553 & 10552 & 4,22 & 6,77 & 14,22 \\
\hline 102 & $\begin{array}{l}\text { Maparajuba/ } \\
\text { maçaranduba }\end{array}$ & $\begin{array}{l}\text { Manilkara amazônica (Huber) } \\
\text { Standley }\end{array}$ & 0,83 & 8,3 & 5,9 & 128,17 & 13533 & 63,55 & 15,20 & 8698 & 9101 & 5,59 & 4,31 & 15,98 \\
\hline 103 & Maragonçalo & Pouteria obscura (Huber) Baehni & 0,64 & 7,4 & 4,4 & 124,45 & 13043 & 61,88 & 14,02 & 10042 & 7767 & 3,33 & 5,10 & 12,65 \\
\hline 104 & Maria-preta & Zizyphus itacaiunensis Fróes & 0,80 & 9,9 & 6,9 & 156,61 & 16181 & 87,48 & 14,51 & 16357 & 14818 & 5,79 & 7,16 & 19,42 \\
\hline 105 & Marupá & Simarouba amara Aubl. & 0,37 & 6,8 & 4,8 & 64,04 & 7257 & 33,05 & 4,41 & 2707 & 1402 & 2,75 & 3,82 & 6,96 \\
\hline
\end{tabular}

FLORESTA, Curitiba, PR, v. 37, n. 3, set./dez. 2007. 


\begin{tabular}{|c|c|c|c|c|c|c|c|c|c|c|c|c|c|c|}
\hline $\mathbf{n}$ & $\begin{array}{l}\text { Nome } \\
\text { usual }\end{array}$ & Nome científico & $\rho_{\text {bas }}$ & $\varepsilon_{\mathrm{r}, 3}$ & $\begin{array}{r}\varepsilon_{\mathrm{r}}, \\
2\end{array}$ & $\mathbf{f}_{\mathbf{M}}$ & $\mathbf{E}_{\mathbf{M 0}}$ & $\mathbf{f}_{\mathrm{c} 0}$ & $\mathbf{E}_{\mathbf{c 9 0}}$ & $\mathbf{f}_{\mathrm{H} 0}$ & $\mathbf{f}_{\mathrm{H} 90}$ & $\begin{array}{r}f_{w t} \\
90 \\
\end{array}$ & $\mathbf{f}_{\mathrm{v} 0}$ & $\mathbf{f}_{\mathrm{s} 0}$ \\
\hline 106 & Matamatá-ci & Eschweilera sp. & 0,81 & 10,7 & 6,0 & 168,18 & 18927 & 86,40 & 17,95 & 14632 & 13631 & - & 8,73 & 20,50 \\
\hline 107 & $\begin{array}{l}\text { Matamatá- } \\
\text { vermelho }\end{array}$ & Eschweilera amara (Aubl.) Ndz. & 0,76 & 10,3 & 4,9 & 141,02 & 14710 & 69,33 & 11,38 & 10895 & 10415 & 4,02 & 7,55 & 14,42 \\
\hline 108 & Melancieira & Alexa grandiflora Ducke & 0,60 & - & - & 109,25 & 13043 & 58,25 & 9,41 & 7532 & 6335 & 4,71 & - & 11,96 \\
\hline 109 & Miraúba & Mouriri callocarpa Ducke & 0,88 & 11,1 & 7,1 & 188,29 & 17162 & 94,44 & 21,97 & 17603 & 17936 & 5,79 & 6,37 & 18,63 \\
\hline 110 & Morototó & $\begin{array}{l}\text { Didymopanax morototoni (Aubl.) } \\
\text { Decne \& Planch. }\end{array}$ & 0,41 & 9,1 & 6,7 & 71,10 & 11082 & 39,72 & 4,51 & 4795 & 3511 & 3,82 & 6,08 & 10,40 \\
\hline 111 & Morototó & $\begin{array}{l}\text { Schefflera morototoni (Aubl.) } \\
\text { Lecne Planch }\end{array}$ & 0,48 & 9,1 & 6,7 & 71,10 & 11082 & 39,72 & 4,51 & 4795 & 3511 & 3,82 & - & 10,40 \\
\hline 112 & Muiracatiara & Astronium lecointei Ducke & 0,79 & 7,6 & 4,6 & 136,41 & 15004 & 82,38 & 13,83 & 8738 & 9591 & 5,39 & 6,18 & 16,77 \\
\hline 113 & Muiracatiara & Astronium ulei Mattick & 0,71 & 8,1 & 4,3 & 115,23 & 13435 & 69,14 & 13,63 & 8140 & 7492 & 5,39 & 7,85 & 16,77 \\
\hline 114 & $\begin{array}{l}\text { Muiracatiara/ } \\
\text { guaritá }\end{array}$ & Astronium gracile Engl. & 0,73 & 8,0 & 5,3 & 130,72 & 5985 & 7012 & 9,81 & 8247 & 7747 & 4,61 & 6,96 & 16,77 \\
\hline 115 & Muirapixuna & Cassia scleroxylon Ducke & 1,01 & 8,2 & 3,6 & 180,83 & 16377 & 96,30 & 22,75 & 14543 & 15279 & 3,24 & 7,26 & 19,91 \\
\hline 116 & Muiratinga & $\begin{array}{l}\text { Maquira sclerophylla (Ducke) C.C. } \\
\text { Berg. }\end{array}$ & 0,57 & - & - & 110,72 & 278 & 60,61 & 8,43 & 8257 & 6178 & 3,92 & - & 11,96 \\
\hline 117 & $\begin{array}{l}\text { Munguba- } \\
\text { terra-firme }\end{array}$ & Eriotheca longipedicellata & 0,45 & 9,8 & 4,5 & 87,77 & 10395 & 47,66 & 5,88 & 6178 & 4599 & 3,53 & - & 8,14 \\
\hline 118 & Mururé & Brosimum acutifolium Huber & 0,67 & 9,1 & 5,0 & 137,49 & 14220 & 76,98 & 14,71 & 14347 & 13504 & 4,12 & 5,39 & 15,98 \\
\hline 119 & Parapará & Jacaranda copaia (Aubl.) D. Don. & 0,31 & 8,2 & 5,4 & 55,11 & 8728 & 30,69 & 3,04 & 3295 & 1883 & 2,84 & - & 5,98 \\
\hline 120 & Parinari & Parinari excelsa Sabine & 0,75 & 10,2 & 5,3 & 145,92 & 16181 & 81,49 & 15,40 & 13082 & 11219 & 5,59 & 5,30 & 14,71 \\
\hline 121 & Pau-amarelo & Euxylophora paraensis Huber & 0,69 & 7,8 & 6,5 & 126,90 & 13729 & 69,43 & 11,96 & 12435 & 10993 & 4,12 & 5,69 & 17,75 \\
\hline 122 & Pau-branco & Drypetes variabilis Uittien & 0,71 & 11,3 & 5,8 & 143,86 & 15887 & 72,47 & 12,36 & 12896 & 11199 & 4,22 & 6,77 & 16,18 \\
\hline 123 & Pau-jacaré & Laetia procera (Poepp.) Eichl. & 0,68 & 12,0 & 5,1 & 120,33 & 15396 & 66,88 & 9,71 & 11229 & 9709 & 6,28 & 7,35 & 16,18 \\
\hline 124 & Pau-santo & Zollernia paraensis Huber & 0,97 & 9,6 & 5,0 & 187,80 & 17848 & 95,52 & 26,97 & 14612 & 14504 & 4,61 & 5,00 & 18,44 \\
\hline 125 & $\begin{array}{l}\text { Pente-de- } \\
\text { macaco }\end{array}$ & Apeiba echinata Gaertn. & 0,36 & - & - & 51,88 & 6669 & 31,77 & 4,41 & 3825 & 2481 & 2,94 & - & 6,18 \\
\hline 126 & Pequiá & Caryocar villosum (Aubl.) Pers. & 0,63 & 8,5 & 4,3 & 99,83 & 11180 & 46,48 & 9,32 & 4864 & 5041 & 5,49 & 5,69 & 12,16 \\
\hline 127 & $\begin{array}{l}\text { Pracuúba-da- } \\
\text { terra-firme }\end{array}$ & Trichilia lecointei Ducke & 0,90 & 8,2 & 4,4 & 157,69 & 16377 & 86,40 & 18,24 & 13062 & 14053 & 5,20 & 6,28 & 18,53 \\
\hline 128 & Preciosa & Aniba canelilla (H.B.К.) Mez. & 0,92 & 8,2 & 6,4 & 183,87 & 17554 & 97,77 & 20,20 & 14710 & 14985 & 2,84 & 5,39 & 18,44 \\
\hline 129 & Quaruba & Vochysia ferruginea Mart. & 0,41 & 10,7 & 5,0 & 66,10 & 7943 & 37,76 & 4,51 & 3158 & - & 2,35 & 3,53 & 7,16 \\
\hline 130 & $\begin{array}{l}\text { Quarubarana } \\
\text { /cedrinho }\end{array}$ & Erisma uncinatum Warm. & 0,46 & 10,0 & 4,3 & 87,48 & 10787 & 49,72 & 5,59 & 5786 & 3864 & 3,04 & 3,73 & 7,85 \\
\hline 131 & $\begin{array}{l}\text { Quaruba- } \\
\text { rosa/quaruba }\end{array}$ & Vochysia guianensis Aubl. & 0,54 & 11,5 & 5,0 & 121,31 & 14024 & 65,31 & 6,77 & 7777 & 6963 & 4,81 & 5,98 & 12,26 \\
\hline 132 & $\begin{array}{l}\text { Quaruba- } \\
\text { rosa/quaruba }\end{array}$ & Vochysia melinonii Beckmann & 0,51 & 10,8 & 4,7 & 97,77 & 11866 & 53,84 & 5,00 & 6914 & 5737 & 4,22 & 6,86 & 11,77 \\
\hline 133 & $\begin{array}{l}\text { Quaruba- } \\
\text { rosa/quaruba }\end{array}$ & Vochysia obidensis (Hub.) Ducke & 0,50 & 10,0 & 3,2 & 100,62 & 12454 & 52,96 & 5,98 & 6718 & 5247 & 4,22 & 5,69 & 10,30 \\
\hline 134 & $\begin{array}{l}\text { Quaruba- } \\
\text { verdad/quarub }\end{array}$ & Vochysia maxima Ducke & 0,49 & 8,8 & 4,0 & 91,20 & 11180 & 47,56 & 5,69 & 5492 & 4717 & 3,43 & 5,00 & 10,00 \\
\hline 135 & Rosadinho & $\begin{array}{l}\text { Micropholis venulosa (Mart. \& } \\
\text { Eichl.) Piérre }\end{array}$ & 0,67 & 9,7 & 4,7 & - & 13925 & 64,92 & 10,00 & 9983 & 7649 & 3,92 & 5,88 & 14,42 \\
\hline 136 & Rosadinho & $\begin{array}{l}\text { Nemaluma anomala (Pires) Pires } \\
\text { (ined.) }\end{array}$ & 0,75 & 9,4 & 6,3 & 162,69 & 15887 & 85,12 & 14,02 & 14347 & 1444 & 4,51 & 6,96 & 18,93 \\
\hline 137 & Roxinho & Peltogyne paniculata Benth. & 0,81 & 8,1 & 5,1 & 187,11 & 17750 & 90,52 & 19,91 & 16181 & 15063 & 2,84 & 4,81 & 18,14 \\
\hline 138 & Seru & $\begin{array}{l}\text { Allantoma lineata (Mart. ex Berg.) } \\
\text { Miers }\end{array}$ & 0,60 & 7,5 & 5,5 & 114,84 & 12749 & 57,96 & 11,28 & 4943 & 5129 & 3,04 & 5,00 & - \\
\hline 139 & Sorva & Malouetia duckei Mgf. & 0,57 & 9,2 & 3,7 & 112,19 & 12651 & 59,23 & 9,71 & 8159 & 5913 & 6,37 & 5,98 & 14,61 \\
\hline 140 & Sucupira & Bowdichia nitida Spruce ex Benth. & 0,85 & 9,0 & 6,0 & 182,11 & 17946 & 92,28 & 15,89 & 14847 & 15200 & 4,12 & 6,57 & 19,02 \\
\hline 141 & Sumaúma & Ceiba pentandra (L.) Gaertn. & 0,29 & 5,5 & 2,7 & 29,32 & 4413 & 23,73 & 1,86 & 2314 & 1510 & 1,86 & - & 3,73 \\
\hline 142 & $\begin{array}{l}\text { Tacacazeiro/ } \\
\text { achichá }\end{array}$ & $\begin{array}{l}\text { Sterculia pilosa Ducke/S. speciosa } \\
\text { K. Sch. }\end{array}$ & 0,53 & - & - & 99 & 1768 & 50,80 & 5,00 & 6208 & 5070 & 4,02 & - & 9,22 \\
\hline 143 & Tachi-branco & Sclerolobium paraense Huber & 0,61 & 8,3 & 4,0 & 123,37 & 13435 & 64,43 & 11,28 & 10728 & 8728 & 4,71 & 6,67 & 17,36 \\
\hline 144 & $\begin{array}{l}\text { Tachi- } \\
\text { pitomba }\end{array}$ & $\begin{array}{l}\text { Sclerolobium chrysophyllum } \\
\text { Poepp. \& Endl. }\end{array}$ & 0,61 & 8,0 & 4,2 & 114,74 & 13533 & 56,39 & 8,83 & 8345 & 6306 & 4,71 & 5,10 & 15,30 \\
\hline 145 & Tachi-pitomba & Sclerolobium poeppigianum Baill. & 0,65 & 8,1 & 4,2 & 128,76 & 13141 & 67,67 & 10,40 & 7983 & 6580 & 4,81 & 6,37 & 17,75 \\
\hline 146 & $\begin{array}{l}\text { Tachi- } \\
\text { preto/tachi }\end{array}$ & Tachigalia cf. myrmecophila Ducke & 0,57 & 7,5 & 5,2 & 130,62 & 12945 & 57,47 & 8,83 & 8600 & 7875 & 3,73 & 6,08 & 14,12 \\
\hline 147 & $\begin{array}{l}\text { Tachi- } \\
\text { vermelho/tac } \\
\text { hirana }\end{array}$ & $\begin{array}{l}\text { Sclerolobium aff. Chrysophyllum } \\
\text { Poepp. \& Endl. }\end{array}$ & 0,62 & - & - & 115,72 & 12258 & 59,13 & 10,98 & 7669 & 5953 & 4,71 & - & 13,44 \\
\hline 148 & Tamaquaré & Caraipa densiflora Mart. & 0,63 & 9,9 & 6,5 & 129,25 & 14808 & 64,82 & 9,12 & 8698 & 6767 & 3,63 & 5,79 & 12,75 \\
\hline 149 & Tanimbuca & Buchenavia capitata Eichl. & 0,70 & 11,6 & 5,9 & 107,68 & 12454 & 59,33 & 12,94 & 11111 & 7296 & 3,43 & 5,30 & 11,38 \\
\hline 150 & Tanimbuca & Buchenavia huberi & 0,79 & 9,1 & 6,0 & 154,95 & 15298 & 83,65 & - & 13317 & 11631 & 4,51 & - & 16,38 \\
\hline 151 & Tatajuba & Bagassa guianensis Aubl. & 0,70 & 5,8 & 4,1 & 222,51 & 11572 & 78,55 & 13,63 & 9875 & 7384 & 5,20 & - & 12,55 \\
\hline 152 & $\begin{array}{l}\text { Tatapiririca/ } \\
\text { maria-preta }\end{array}$ & Tapirira guianensis Aubl. & 0,50 & - & - & 83,06 & 10885 & 45,31 & 6,77 & 5345 & 3962 & 5,39 & - & 10,69 \\
\hline
\end{tabular}




\begin{tabular}{|c|c|c|c|c|c|c|c|c|c|c|c|c|c|c|}
\hline $\mathbf{n}$ & $\begin{array}{l}\text { Nome } \\
\text { usual }\end{array}$ & Nome científico & $\rho_{\text {bas }}$ & $\varepsilon_{\mathrm{r}, 3}$ & $\begin{array}{r}\varepsilon_{\mathrm{r}} \\
2\end{array}$ & $\mathbf{f}_{\mathbf{M}}$ & $\mathbf{E}_{\mathbf{M 0}}$ & $\mathbf{f}_{\mathrm{c} 0}$ & $\mathbf{E}_{\mathbf{c 9 0}}$ & $\mathbf{f}_{\mathrm{HO}}$ & $\mathbf{f}_{\mathrm{H} 90}$ & $\begin{array}{r}\mathbf{f}_{\mathrm{wt}} \\
90\end{array}$ & $\mathbf{f}_{\mathrm{v} 0}$ & $\mathbf{f}_{\mathrm{s} 0}$ \\
\hline 153 & Tauari & $\begin{array}{l}\text { Couratari oblongifolia Ducke \& R. } \\
\text { Knuth }\end{array}$ & 0,50 & 6,6 & 4,2 & 83,06 & 10885 & 44,62 & 6,28 & 5776 & 4599 & 3,73 & 4,81 & 10,40 \\
\hline 154 & Tauari & Couratari guianensis & 0,52 & 6,1 & 3,6 & 104,05 & 11474 & 53,94 & 7,75 & 6521 & 5060 & 4,12 & - & 10,20 \\
\hline 155 & Tauari & Couratari stellata A. C. Smith & 0,65 & 7,8 & 5,8 & 134,06 & 14318 & 69,14 & 11,57 & 8777 & 6963 & 4,51 & - & 12,85 \\
\hline 156 & Tento & Ormosia paraensis Ducke & 0,67 & 10,3 & 4,7 & 122,78 & 14612 & 70,61 & 10,59 & 8659 & 8208 & 3,63 & 4,90 & 12,75 \\
\hline 157 & Tuturubá & Pouteria oblanceolata Pires & 0,79 & 8,9 & 5,2 & 153,18 & 15396 & 77,86 & 13,73 & 13200 & 13004 & 4,02 & 5,00 & 15,98 \\
\hline 158 & $\begin{array}{l}\text { Uchi- } \\
\text { liso/uchi }\end{array}$ & Endopleura uchi (Huber) Cuatr. & 0,78 & 9,6 & 6,4 & 153,67 & 15298 & 74,82 & 13,04 & 13111 & 10385 & 6,18 & 4,71 & 18,73 \\
\hline 159 & Uchirana & Vantanea parviflora Lam. & 0,86 & 10,3 & 8,2 & 169,85 & 17554 & 86,40 & 15,20 & 15436 & 13111 & 4,71 & 5,69 & 17,75 \\
\hline 160 & $\begin{array}{l}\text { Ucuúba-da- } \\
\text { terra-firme }\end{array}$ & Virola michellii Heckel & 0,50 & - & - & 95,32 & 11866 & 51,19 & 4,90 & 6580 & 4629 & 4,81 & - & 9,90 \\
\hline 161 & Ucuubarana & $\begin{array}{l}\text { Osteophloeum platyspermum (A. } \\
\text { DC.) Warb. }\end{array}$ & 0,50 & 7,4 & 3,6 & 88,06 & 12356 & 45,99 & 5,00 & 4354 & 3432 & 3,53 & 4,22 & 9,02 \\
\hline 162 & Ucuubarana & Iryanthera grandis Ducke & 0,63 & - & - & 106,40 & 12454 & 59,72 & 8,14 & 5178 & 5315 & 4,61 & - & 9,61 \\
\hline 163 & $\begin{array}{l}\text { Urucu-da- } \\
\text { mata }\end{array}$ & Bixa arborea Huber & 0,32 & - & - & 54,43 & 7551 & 35,79 & 3,92 & 3883 & 1942 & 2,35 & - & 6,28 \\
\hline
\end{tabular}

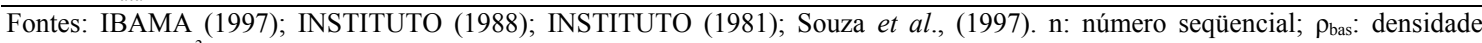
básica, em g.cm $\mathrm{cm}^{-3} ; \varepsilon_{\mathrm{r}, 3}$ : contração tangencial, em $\% ; \varepsilon_{\mathrm{r}, 2}$ : contração radial, em $\%$; $\mathrm{f}_{\mathrm{M}}$ : módulo de ruptura à flexão estática, em MPa; $\mathrm{E}_{\mathrm{M} 0}$ : módulo de elasticidade à flexão estática, em $\mathrm{MPa} ; \mathrm{f}_{\mathrm{c} 0}$ : resistência à compressão paralela às fibras, em $\mathrm{MPa} ; \mathrm{E}_{\mathrm{c} 90}$ : resistência no limite proporcional à compressão perpendicular às fibras, em $\mathrm{MPa} ; \mathrm{f}_{\mathrm{H} 0}$ : dureza Janka paralela às fibras, em $\mathrm{N}$; $\mathrm{f}_{\mathrm{H} 90}$ : dureza Janka transversal às fibras, em $\mathrm{N} ; \mathrm{f}_{\mathrm{w} t 90}$ : resistência à tração perpendicular às fibras, em $\mathrm{MPa} ; \mathrm{f}_{\mathrm{v} 0}$ : resistência ao fendilhamento, em $\mathrm{MPa}$; $\mathrm{f}_{\mathrm{s} 0}$ : resistência ao cisalhamento, em $\mathrm{MPa}$.

\section{Software estatístico}

As análises estatísticas das relações funcionais das PFMM foram realizadas através do programa computacional Statistical Analysis System - SAS (SAS INSTITUTE INC., 1993).

\section{Correlações}

As correlações das PFMM deste estudo são expressas pelo coeficiente de correlação linear de Pearson $(\alpha)$. $\mathrm{O} \alpha$ é adimensional e dado pela expressão:

$$
\alpha=\frac{\frac{1}{n-1} \sum_{i=1}^{n}\left(x_{i}-\mu_{x}\right)\left(y_{i}-\mu_{y}\right)}{\sigma_{x} \sigma_{y}}
$$

Onde: $\quad \alpha=$ coeficiente de correlação linear de Pearson;

$\mathrm{n}=$ número de observações de cada variável;

$\mathrm{x}_{\mathrm{i}}$ e $\mathrm{y}_{\mathrm{i}}=$ valores i-ésimos das variáveis $\mathrm{x}$ e $\mathrm{y}$, respectivamente;

$\mu_{\mathrm{x}}$ e $\mu_{\mathrm{y}}=$ médias das variáveis $\mathrm{x}$ e $\mathrm{y}$, respectivamente;

$\sigma_{\mathrm{x}}$ e $\sigma_{\mathrm{y}}=$ desvios padrão das variáveis $\mathrm{x}$ e $\mathrm{y}$, respectivamente.

Para cada propriedade foi determinado o coeficiente de correlação médio $\left(\alpha_{\mathrm{m}}\right)$, o qual é calculado pela razão entre a soma dos $\alpha$ de cada propriedade com o das demais e o número total de propriedades menos um, ou seja, onze. Embora sem muita validade estatística, os $\alpha_{\mathrm{m}}$ são aqui considerados índices que espelham o quanto as PFMM correlacionam-se umas com as outras em termos totais. É dado por:

$$
\alpha_{\mathrm{m}}=\frac{\sum_{\mathrm{i}=1}^{\mathrm{n}-1} \alpha \mathrm{p}_{\mathrm{i}}}{\mathrm{n}-1}
$$

Onde: $\quad \alpha \mathrm{m}=$ coeficiente de correlação médio;

$\alpha$ pi = coeficientes de correlação linear da i-ésima PFMM;

$\mathrm{n}=$ número de propriedades.

\section{Análises de regressão (equações de regressão)}

Foi utilizado $\rho_{\text {bas }}$ como variável independente para o ajuste das equações de regressão de todas as outras propriedades. Essa opção foi adotada em razão de sua já destacada importância, e também por ser a propriedade mais freqüente (menos ausente) nas fontes de dados consultadas. Além disso, $\rho_{\text {bas }}$ é a 
propriedade que apresenta um dos maiores $\alpha_{\mathrm{m}}$, o que indica a sua adequação para fins de análises de regressão e, assim sendo, para estimativas. Vale citar que na literatura encontram-se vários exemplos do emprego da $\rho_{\text {bas }}$ como variável independente em equações de regressão para estimativas de outras PFMM (BODIG; JAYNE, 1982; CHIMELO, 1980; NASCIMENTO et al., 1997; ROCHA, 1994; USDA, 1999).

Os cálculos foram feitos utilizando o procedimento Guided Data Analysis ("Análise de Dados Guiada") do programa SAS. Tal procedimento, além da análise de regressão propriamente dita, proporciona a avaliação de suposições estatísticas dos dados sob análise, permitindo identificar e corrigir as imperfeições ou violações estatísticas, tais como: a) escala dos dados da variável a ser estimada (efetua transformação logarítmica, raiz quadrada, inversa, etc.); b) indicação do modelo de equação mais apropriado (linear, quadrático ou cúbico); c) identificação e exclusão de outliers (valores discrepantes).

O procedimento Guided Data Analysis, ainda que, em algumas situações, indique modelos de equações de regressão mais complexos quando comparados aos exemplos encontrados na literatura, tem a vantagem de aumentar, devido às citadas correções estatísticas nos dados sob análise, a eficiência dessas equações para fins de estimativas.

Como critério para se saber se a equação ajustada representou adequadamente a relação funcional entre as variáveis, usou-se o coeficiente de determinação $\left(\mathrm{R}^{2}\right)$.

\section{RESULTADOS E DISCUSSÃO}

\section{Estatística simples da amostra}

Os parâmetros estatísticos simples dos dados da amostra são mostrados na tabela 4.

A variação do número de dados verificada se deve à falta de informação nas fontes consultadas (por exemplo: para $\rho_{\text {bas }}$, duas espécies não apresentaram a informação dessa propriedade, o que totalizou 161 observações efetivas).

$\mathrm{O}$ coeficiente de variação percentual $(\mathrm{Cv} \%)$ revelou uma variabilidade acentuada das propriedades, sendo que o menor $\mathrm{Cv} \%$ foi o de $\varepsilon_{\mathrm{r}, 3}(17,9 \%)$ e o maior o de $\mathrm{f}_{\mathrm{H} 90}(52,4 \%)$. Sob a ótica de representação da variação natural existente entre as espécies de madeira, a alta variabilidade dos dados da amostra utilizada pode ser interpretada como um aspecto positivo, pois significa que abrangeu espécies com boa proximidade da amplitude natural que as PFMM podem assumir, o que pode ser verificado pelos seus valores máximos e mínimos.

Tabela 4. Parâmetros estatísticos simples dos dados da amostra das propriedades físicas e mecânicas das madeiras (PFMM) do estudo.

Table 4. Simple statistical parameters of the data of the sample of the physical and mechanical properties of wood (PFMM) of the study.

\begin{tabular}{|c|c|c|c|c|c|c|}
\hline Propriedade & $\mathbf{n}$ & Média & Desvio Padrão & CV\% & Valor mínimo & Valor máximo \\
\hline$\rho_{\text {bas }}$ & 161 & 0,63 & 0,15 & 24,3 & 0,29 & 1,01 \\
\hline$\varepsilon_{\mathrm{r}, 3}$ & 140 & 8,78 & 1,57 & 17,9 & 4,50 & 12,60 \\
\hline$\varepsilon_{\mathrm{r}, 2}$ & 140 & 4,92 & 1,10 & 22,3 & 2,40 & 8,20 \\
\hline$f_{M}$ & 158 & 120,25 & 36,95 & 30,7 & 29,32 & 222,51 \\
\hline $\mathrm{E}_{\mathrm{M} 0}$ & 159 & 13318 & 2809 & 21,1 & 4413 & 18927 \\
\hline $\mathrm{f}_{\mathrm{c} 0}$ & 159 & 63,67 & 17,63 & 27,7 & 23,73 & 100,13 \\
\hline $\mathrm{E}_{\mathrm{c} 90}$ & 154 & 10,56 & 5,00 & 47,3 & 1,86 & 26,97 \\
\hline $\mathrm{f}_{\mathrm{H} 0}$ & 158 & 8773,8 & 3958,4 & 45,1 & 2079 & 17603 \\
\hline $\mathrm{f}_{\mathrm{H} 90}$ & 157 & 7730,15 & 4048,96 & 52,4 & 1196 & 17936 \\
\hline $\mathrm{f}_{\mathrm{wt} 90}$ & 154 & 4,10 & 1,09 & 26,6 & 1,86 & 7,65 \\
\hline$f_{v 0}$ & 115 & 5,71 & 1,33 & 23,3 & 2,94 & 8,83 \\
\hline $\mathrm{f}_{\mathrm{s} 0}$ & 157 & 13,10 & 4,24 & 32,4 & 3,73 & 23,14 \\
\hline
\end{tabular}

$\rho_{\text {bas }}$ : densidade básica, em g.cm ${ }^{-3} ; \varepsilon_{\mathrm{r}, 3}$ : contração tangencial, em $\% ; \varepsilon_{\mathrm{r}, 2}$ : contração radial, em $\%$; $\mathrm{f}_{\mathrm{M}}$ : módulo de ruptura à flexão estática, em $\mathrm{MPa} ; \mathrm{E}_{\mathrm{M} 0}$ : módulo de elasticidade à flexão estática, em $\mathrm{MPa} ; \mathrm{f}_{\mathrm{c} 0}$ : resistência à compressão paralela às fibras, em $\mathrm{MPa}$; $\mathrm{E}_{\mathrm{c} 90}$ : resistência no limite proporcional à compressão perpendicular às fibras, em $\mathrm{MPa} ; \mathrm{f}_{\mathrm{H} 0}$ : dureza Janka paralela às fibras, em $\mathrm{N}$; $\mathrm{f}_{\mathrm{H} 90}$ : dureza Janka transversal às fibras, em $\mathrm{N} ; \mathrm{f}_{\mathrm{w} t 90}$ : resistência à tração perpendicular às fibras, em $\mathrm{MPa}$; $\mathrm{f}_{\mathrm{v} 0}$ : resistência ao fendilhamento, em MPa; $\mathrm{f}_{\mathrm{s} 0}$ : resistência ao cisalhamento, em $\mathrm{MPa} ; \mathrm{n}$ : número de dados; $\mathrm{Cv} \%$ : coeficiente de variação percentual. 


\section{Correlações}

A matriz dos coeficientes de correlação $(\alpha)$ das PFMM é apresentada na tabela 5.

Tabela 5. Matriz dos coeficientes de correlação $(\alpha)$ das propriedades físicas e mecânicas das madeiras (PFMM) do estudo.

Table 5. Matrix of the correlation coefficients $(\alpha)$ of the physical and mechanical properties of wood (PFMM) of the study.

\begin{tabular}{|c|c|c|c|c|c|c|c|c|c|c|c|c|}
\hline Propriedade & $\rho_{\text {bas }}$ & $\varepsilon_{\mathrm{r}, 3}$ & $\varepsilon_{\mathrm{r}, 2}$ & $\mathbf{f}_{M}$ & $\mathbf{E}_{\mathbf{M} 0}$ & $\mathbf{f}_{\mathrm{c} 0}$ & $\mathbf{E}_{\mathrm{c90}}$ & $\mathbf{f}_{\mathrm{H} 0}$ & $f_{\mathrm{H} 90}$ & $\mathbf{f}_{\mathrm{wt} 90}$ & $\mathbf{f}_{\mathrm{v} 0}$ & $\mathbf{f}_{\mathrm{s} 0}$ \\
\hline$\rho_{\text {bas }}$ & $\begin{array}{c}1 \\
(161)\end{array}$ & - & - & - & - & - & - & - & - & - & - & - \\
\hline$\varepsilon_{\mathrm{r}, 3}$ & $\begin{array}{c}0,3634^{* * *} \\
(138)\end{array}$ & $\begin{array}{c}1 \\
(140)\end{array}$ & - & - & - & - & - & - & - & - & - & - \\
\hline$\varepsilon_{\mathrm{r}, 2}$ & $\begin{array}{c}0,4279^{* *} \\
(138)\end{array}$ & $\begin{array}{c}0,6387^{* *} \\
(140)\end{array}$ & $\begin{array}{c}1 \\
(140)\end{array}$ & - & - & - & - & - & - & - & - & - \\
\hline$f_{M}$ & $\begin{array}{c}0,9224 * * \\
(158)\end{array}$ & $\begin{array}{c}0,3572^{* *} \\
(135)\end{array}$ & $\begin{array}{c}0,4440^{* *} \\
(135)\end{array}$ & $\begin{array}{c}1 \\
(158)\end{array}$ & - & - & - & - & - & - & - & - \\
\hline $\mathrm{E}_{\mathrm{M} 0}$ & $\begin{array}{c}0,8917 * * \\
(159)\end{array}$ & $\begin{array}{c}0,4991^{* *} \\
(136)\end{array}$ & $\begin{array}{c}0,5068^{* *} \\
(136)\end{array}$ & $\begin{array}{c}0,9061^{* *} \\
(158)\end{array}$ & $\begin{array}{c}1 \\
(159)\end{array}$ & - & - & - & - & - & - & - \\
\hline $\mathrm{f}_{\mathrm{c} 0}$ & $\begin{array}{c}0,9416^{* *} \\
(159)\end{array}$ & $\begin{array}{c}0,3894^{* *} \\
(136)\end{array}$ & $\begin{array}{c}0,4394 * * \\
(136)\end{array}$ & $\begin{array}{c}0,9597 * * \\
(158)\end{array}$ & $\begin{array}{c}0,9238^{* *} \\
(159)\end{array}$ & $\begin{array}{c}1 \\
(159)\end{array}$ & - & - & - & - & - & - \\
\hline $\mathrm{E}_{\mathrm{c} 90}$ & $\begin{array}{c}0,9124 * * \\
(154)\end{array}$ & $\begin{array}{c}0,2155^{*} \\
(133)\end{array}$ & $\begin{array}{c}0,3213^{* *} \\
(133)\end{array}$ & $\begin{array}{c}0,8821^{* *} \\
(153)\end{array}$ & $\begin{array}{c}0,7733^{* *} \\
(154)\end{array}$ & $\begin{array}{c}0,8907^{* *} \\
(154)\end{array}$ & $\begin{array}{c}1 \\
(154)\end{array}$ & - & - & - & - & - \\
\hline $\mathrm{f}_{\mathrm{H} 0}$ & $\begin{array}{c}0,8769 * * \\
(158)\end{array}$ & $\begin{array}{c}0,4030^{* * *} \\
(135)\end{array}$ & $\begin{array}{c}0,4493 * * \\
(135)\end{array}$ & $\begin{array}{c}0,8864 * * \\
(157)\end{array}$ & $\begin{array}{c}0,8428^{* *} \\
(158)\end{array}$ & $\begin{array}{c}0,9061^{* *} \\
(158)\end{array}$ & $\begin{array}{c}0,8572 * * \\
(153)\end{array}$ & $\begin{array}{c}1 \\
(158)\end{array}$ & - & - & - & - \\
\hline $\mathrm{f}_{\mathrm{H} 90}$ & $\begin{array}{c}0,9207 * * \\
(157)\end{array}$ & $\begin{array}{c}0,3926^{* *} \\
(134)\end{array}$ & $\begin{array}{c}0,4388^{* *} \\
(134)\end{array}$ & $\begin{array}{c}0,9019^{* *} \\
(156)\end{array}$ & $\begin{array}{c}0,8679 * * \\
(157)\end{array}$ & $\begin{array}{c}0,9290^{* *} \\
(157)\end{array}$ & $\begin{array}{c}0,8960^{* * *} \\
(152)\end{array}$ & $\begin{array}{c}0,9724^{* *} \\
(156)\end{array}$ & $\begin{array}{c}1 \\
(157)\end{array}$ & - & - & - \\
\hline$f_{w t 90}$ & $\begin{array}{c}0,5087^{* *} \\
(154)\end{array}$ & $\begin{array}{c}0,2441^{*} \\
(131)\end{array}$ & $\begin{array}{c}0,0733^{\mathrm{ns}} \\
(131)\end{array}$ & $\begin{array}{c}0,5024 * * \\
(153)\end{array}$ & $\begin{array}{c}0,4717 * * \\
(154)\end{array}$ & $\begin{array}{c}0,4946^{* *} \\
(154)\end{array}$ & $\begin{array}{c}0,4481^{* * *} \\
(150)\end{array}$ & $\begin{array}{c}0,4887 * * \\
(153)\end{array}$ & $\begin{array}{c}0,4717^{* *} \\
(152)\end{array}$ & $\begin{array}{c}1 \\
(154)\end{array}$ & - & - \\
\hline$f_{v 0}$ & $\begin{array}{c}0,6114^{* *} \\
(115)\end{array}$ & $\begin{array}{c}0,3477 * * \\
(115)\end{array}$ & $\begin{array}{c}0,1849^{*} \\
(115)\end{array}$ & $\begin{array}{c}0,6185^{* *} \\
(114)\end{array}$ & $\begin{array}{c}0,6186^{* *} \\
(115)\end{array}$ & $\begin{array}{c}0,6213^{* *} \\
(115)\end{array}$ & $\begin{array}{c}0,5211^{* *} \\
(114)\end{array}$ & $\begin{array}{c}0,5813^{* *} \\
(114)\end{array}$ & $\begin{array}{c}0,5842^{* *} \\
(113)\end{array}$ & $\begin{array}{c}0,6849^{* *} \\
(113)\end{array}$ & $\begin{array}{c}1 \\
(115)\end{array}$ & - \\
\hline$f_{s 0}$ & $\begin{array}{c}0,8820^{* * *} \\
(157)\end{array}$ & $\begin{array}{c}0,3109^{* *} \\
(134)\end{array}$ & $\begin{array}{c}0,3393 * * \\
(134)\end{array}$ & $\begin{array}{c}0,8613^{* *} \\
(156)\end{array}$ & $\begin{array}{c}0,8443^{* *} \\
(157)\end{array}$ & $\begin{array}{c}0,8777^{* *} \\
(157)\end{array}$ & $\begin{array}{c}0,8342 * * \\
(152)\end{array}$ & $\begin{array}{c}0,9032^{* *} \\
(156)\end{array}$ & $\begin{array}{c}0,9110^{* *} \\
(155)\end{array}$ & $\begin{array}{c}0,6324 * * \\
(153)\end{array}$ & $\begin{array}{c}0,7191^{* *} \\
(114)\end{array}$ & $\begin{array}{c}1 \\
(157)\end{array}$ \\
\hline
\end{tabular}

$\rho_{\text {bas: }}$ : densidade básica, em g.cm ${ }^{-3} ; \varepsilon_{\mathrm{r}, 3}$ : contração tangencial, em $\% ; \varepsilon_{\mathrm{r}, 2}$ : contração radial, em $\% ; \mathrm{f}_{\mathrm{M}}$ : módulo de ruptura à flexão estática, em $\mathrm{MPa} ; \mathrm{E}_{\mathrm{M} 0}$ : módulo de elasticidade à flexão estática, em $\mathrm{MPa} ; \mathrm{f}_{\mathrm{c} 0}$ : resistência à compressão paralela às fibras, em $\mathrm{MPa}$; $\mathrm{E}_{\mathrm{c} 90}$ : resistência no limite proporcional à compressão perpendicular às fibras, em $\mathrm{MPa} ; \mathrm{f}_{\mathrm{H} 0}$ : dureza Janka paralela às fibras, em $\mathrm{N}$; $\mathrm{f}_{\mathrm{H} 90}$ : dureza Janka transversal às fibras, em $\mathrm{N} ; \mathrm{f}_{\mathrm{w} t 90}$ : resistência à tração perpendicular às fibras, em $\mathrm{MPa} ; \mathrm{f}_{\mathrm{v} 0}$ : resistência ao fendilhamento, em MPa; $\mathrm{f}_{\mathrm{s} 0}$ : resistência ao cisalhamento, em $\mathrm{MPa}$; entre parênteses, o número de pares de dados utilizados para o cálculo do $\alpha$;*: $\alpha$ significativo ao nível de $1 \%$ de probabilidade $(\mathrm{P}<0,01$ de $\mathrm{Ho})$; $: \alpha$ significativo ao nível de $5 \%$ de probabilidade $(\mathrm{P}<0,05$ de Ho); ns: $\alpha$ não significativo $(\mathrm{P}>0,05$ de Ho)

Para a maior parte das propriedades houve expressiva correlação. O máximo valor de $\alpha$ encontrado foi o de $\mathrm{f}_{\mathrm{H} 0} v s$. $\mathrm{f}_{\mathrm{H} 90}(0,9724)$, e o mínimo valor, estatisticamente significante, foi o de $\varepsilon_{\mathrm{r}, 2} v s . \mathrm{f}_{\mathrm{v} 0}$ $(0,1849)$, mostrando quase inexistência de correlação. A correlação menos expressiva, além de ser a única sem significância estatística, foi a de $\varepsilon_{\mathrm{r}, 2} v s$. $\mathrm{f}_{\mathrm{wt} 90}(0,0733)$. Em termos de propriedades físicas versus mecânicas, o maior valor de $\alpha$ foi o de $\rho_{\text {bas }} v s . \mathrm{f}_{\mathrm{c} 0}(0,9416)$, e o menor, estatisticamente significante, foi o mencionado para $\varepsilon_{\mathrm{r}, 2} v s . \mathrm{f}_{\mathrm{v} 0}$.

Os $\alpha$ da densidade básica com as propriedades mecânicas, que variaram de $0,5087\left(\rho_{\text {bas }} v s . f_{w t 90}\right)$ a $0,9416\left(\rho_{\text {bas }} v s . \mathrm{f}_{\mathrm{c} 0}\right)$, mostraram-se, com exceção de $\mathrm{f}_{\mathrm{w} t 90}$ e $\mathrm{f}_{\mathrm{v} 0}$, bastante altos, todos acima de 0,87 . Esses resultados são compatíveis com os descritos por Paula et al. (1986) e Nascimento (1993), que encontraram, por regressão linear simples, para madeiras amazônicas secas a um teor de umidade (t.u.), valores do $R^{2}$, entre $\rho_{\text {bas }}$ e as propriedades mecânicas $f_{M}, E_{M 0}, f_{c 0}, E_{c 90}$ e $f_{s 0}$, variando de 0,58 a 0,77 e 0,56 a 0,88 , respectivamente. Vale lembrar que o valor do $\mathrm{R}^{2}$ em regressão linear simples é o quadrado do valor do $\alpha$.

A figura 1 representa os valores dos coeficientes de correlação médios $\left(\alpha_{m}\right)$ de cada propriedade, fornecendo uma visualização resumida dessas relações em termos totais.

As três propriedades com os maiores índices de $\alpha_{\mathrm{m}}$ foram $\mathrm{f}_{\mathrm{c} 0}, \mathrm{f}_{\mathrm{H} 90}$ e $\rho_{\text {bas }}$, sendo que $\mathrm{f}_{\mathrm{c} 0}$ apresentou o máximo valor $(0,7612)$. As três com os menores índices foram $\varepsilon_{\mathrm{r}, 3}, \varepsilon_{\mathrm{r}, 2}$, e $f_{\mathrm{wt} 90}$, sendo que $\varepsilon_{\mathrm{r}, 3}$ apresentou o mínimo valor $(0,3783)$. As propriedades $\mathrm{f}_{\mathrm{M}}, \mathrm{E}_{\mathrm{M} 0}, \mathrm{f}_{\mathrm{H} 0}$ e $\mathrm{f}_{\mathrm{s} 0}$ apresentaram significativos valores de $\alpha_{\mathrm{m}}$, todos na casa dos 0,74 . Já $\mathrm{E}_{\mathrm{c} 90}$ e $\mathrm{f}_{\mathrm{v} 0}$ revelaram índices moderados $(0,6865$ e 0,5539 , respectivamente).

$\mathrm{O}$ resultado do $\alpha_{\mathrm{m}}$ de $\rho_{\text {bas }}(0,7508)$ comprova que a massa específica da madeira tem estreitas relações com as outras propriedades, principalmente as mecânicas. 
Pelo critério $\alpha_{\mathrm{m}}$, os resultados mostram ser possível relacionar de maneira próxima à proporcionalidade (isto é, as variações do valor de uma propriedade correspondem aproximadamente às mesmas variações de outra) as PFMM melhor correlacionadas, quais sejam: $f_{c 0}, f_{H 90}, \rho_{\text {bas }}, f_{M}, f_{H 0}, E_{M 0}, f_{s 0}$ e $\mathrm{E}_{\mathrm{c} 90}$. Tal possibilidade, no entanto, não é permitida para $\varepsilon_{\mathrm{r}, 3}, \varepsilon_{\mathrm{r}, 2}, \mathrm{f}_{\mathrm{w} t 90}$ e $\mathrm{f}_{\mathrm{v} 0}$.

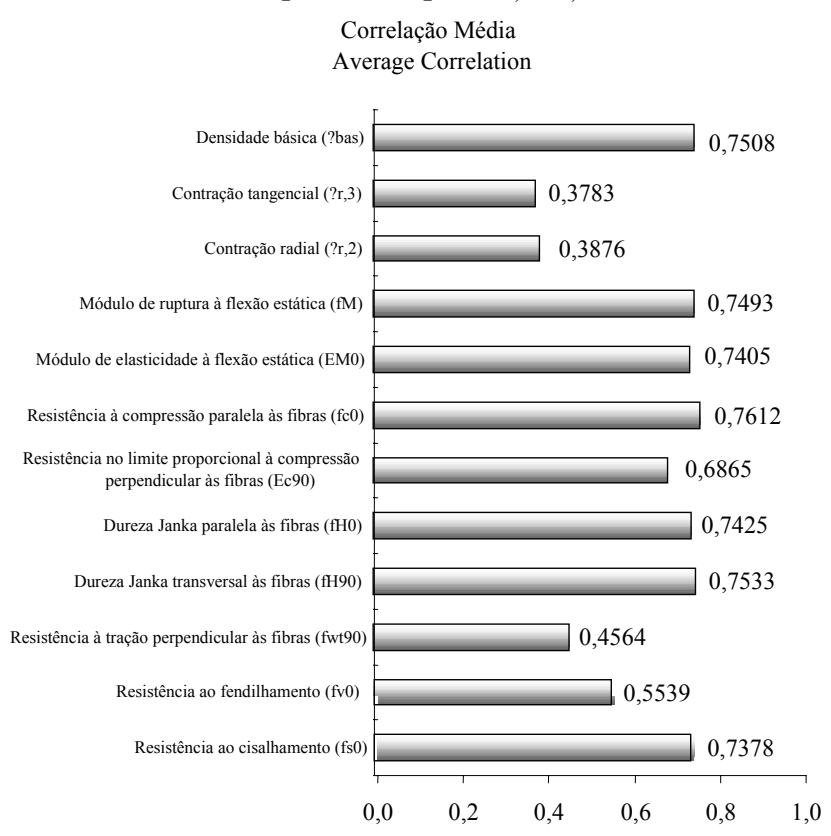

Figura 1. Coeficientes de correlação médios $(\alpha \mathrm{m})$ das propriedades físicas e mecânicas das madeiras (PFMM) do estudo.

Figure 1. Averages correlation coefficients $(\alpha \mathrm{m})$ of the physical and mechanical properties of wood (PFMM) of the study.

$\mathrm{O} \alpha_{\mathrm{m}}$ de $\mathrm{f}_{\mathrm{v} 0}(0,5539)$ recomenda cautela para associá-la às outras PFMM, especialmente aquelas com as correlações mais baixas (a exemplo de $\varepsilon_{\mathrm{r}, 2}, \varepsilon_{\mathrm{r}, 3}$ e $\mathrm{E}_{\mathrm{c} 90}$ ).

Os $\alpha_{\mathrm{m}}$ de $\varepsilon_{\mathrm{r}, 3}(0,3783), \varepsilon_{\mathrm{r}, 2}(0,3876)$ e $\mathrm{f}_{\mathrm{wt} 90}(0,4564)$ indicam, especialmente os dois primeiros, inconveniência do uso dessas propriedades para caracterizar ou inferir valores à grande maioria das outras PFMM. No caso de $f_{w t 90}$, fazem-se ressalvas para $f_{v 0}(\alpha=0,6849)$ e $f_{s 0}(\alpha=0,6324)$.

Observou-se que, embora os seus $\alpha_{\mathrm{m}}$ tenham se mostrado bastante fracos, o $\alpha$ de $\varepsilon_{\mathrm{r}, 3}$ e $\varepsilon_{\mathrm{r}, 2}$ é relativamente significativo $(0,6387)$, sendo o mais alto entre as propriedades de contração e as outras, o que permite, de modo cauteloso, correlacioná-las.

Chimelo (1980), estudando 32 espécies amazônicas na condição verde (t.u. saturado), através de regressão linear, obteve um $\mathrm{R}^{2}$ de 0,02 e 0,09 , respectivamente, nas relações entre $\varepsilon_{\mathrm{r}, 3}$ e $\varepsilon_{\mathrm{r}, 2}$ com $\rho_{\mathrm{bas}}$, contra $R^{2}$ variando de 0,63 a 0,78 nas relações entre propriedades mecânicas $f_{M}, E_{M 0}$, $E_{c 90}$ e $f_{s 0}$ com $\rho_{\text {bas }}$. Rocha (1994), estudando 150 espécies amazônicas, tanto na condição verde como no t.u. de $12 \%$, também por regressão linear, obteve $\mathrm{R}^{2}$ de 0,08 para $\rho_{\text {bas }} v s . \varepsilon_{\mathrm{r}, 3}$ e 0,22 para $\rho_{\mathrm{bas}} v s . \varepsilon_{\mathrm{r}, 2}$, enquanto que os $\mathrm{R}^{2}$ de $\rho_{\text {bas }}$ com propriedades mecânicas $\mathrm{f}_{\mathrm{M}}, \mathrm{E}_{\mathrm{M} 0}, \mathrm{E}_{\mathrm{c} 90}$ e $\mathrm{f}_{\mathrm{s} 0}$ variaram entre 0,58 e 0,77.

Neste estudo, os baixos valores de $\alpha$ das propriedades de contração com $\rho_{\text {bas, }}$ que foram de $0,3634\left(\varepsilon_{\mathrm{r}, 3} v s . \rho_{\mathrm{bas}}\right)$ e $0,4279\left(\varepsilon_{\mathrm{r}, 2} v s . \rho_{\text {bas }}\right)$, bem como os altos $\alpha$ entre $\rho_{\text {bas }}$ e as propriedades $\mathrm{f}_{\mathrm{M}}, \mathrm{E}_{\mathrm{M} 0}, \mathrm{E}_{\mathrm{c} 90} \mathrm{e}$ $\mathrm{f}_{\mathrm{s} 0}$, que variaram entre $0,8820\left(\rho_{\text {bas }}\right.$ vs. $\left.\mathrm{f}_{\mathrm{s} 0}\right)$ e $0,9224\left(\rho_{\text {bas }} v s . \mathrm{f}_{\mathrm{M}}\right)$, confirmam as pesquisas de Chimelo (1980) e Rocha (1994), demonstrando que, de fato, as relações de $\rho_{\mathrm{bas}}$ com $\varepsilon_{\mathrm{r}, 3}$ e $\varepsilon_{\mathrm{r}, 2}$ são fracas, enquanto que com as propriedades mecânicas $\mathrm{f}_{\mathrm{M}}, \mathrm{E}_{\mathrm{M} 0}, \mathrm{E}_{\mathrm{c} 90}$ e $\mathrm{f}_{\mathrm{s} 0}$ são, ao contrário, bastante consistentes.

\section{Análises de regressão (equações de regressão)}

Para as análises de regressão, os dados básicos foram submetidos ao procedimento Guided Data Analysis do programa SAS, tendo $\rho_{\text {bas }}$ como variável independente em relação às outras PFMM. De acordo com as características dos dados (distribuição, escala, etc.), a análise guiada do SAS indicou a 
necessidade de transformação das variáveis dependentes, identificou e eliminou os outliers e apontou o modelo de equação mais apropriado a cada conjunto de observações (Tabela 6).

Tabela 6. Resultados das análises de suposições estatísticas dos dados para as equações de regressão utilizando o SAS Guided Data Analysis.

Table 6. Results of the analysis of statistical suppositions of the data for the regression equations using the SAS Guided Dates Analysis.

\begin{tabular}{|c|c|c|c|c|}
\hline Propriedade & $\begin{array}{c}\text { Transformação da } \\
\text { variável dependente }\end{array}$ & $\begin{array}{c}\text { Outliers } \\
\text { eliminados }\end{array}$ & $\begin{array}{c}\text { Modelo de equação } \\
\text { apropriado }\end{array}$ & Equação a ajustar \\
\hline$\varepsilon_{\mathrm{r}, 3}$ & sem transformação & 0 & Linear & $\varepsilon_{\mathrm{r}, 3}=\mathrm{a}+\mathrm{b} \rho_{\mathrm{bas}}$ \\
\hline$\varepsilon_{\mathrm{r}, 2}$ & sem transformação & 0 & Cúbica & $\varepsilon_{\mathrm{r}, 2}=\mathrm{a}+\mathrm{b} \rho_{\mathrm{bas}}+\mathrm{c} \rho_{\mathrm{bas}}{ }^{2}+\mathrm{d} \rho_{\mathrm{bas}}{ }^{3}$ \\
\hline$f_{\mathrm{M}}$ & $\sqrt{f_{M}}$ & 2 & Quadrática & $\sqrt{f_{\mathrm{M}}}=\mathrm{a}+\mathrm{b} \rho_{\mathrm{bas}}+\mathrm{c} \rho_{\mathrm{bas}}^{2}$ \\
\hline $\mathrm{E}_{\mathrm{M} 0}$ & sem transformação & 0 & Quadrática & $\mathrm{E}_{\mathrm{M} 0}=\mathrm{a}+\mathrm{b} \rho_{\text {bas }}+\mathrm{c} \rho_{\text {bas }}^{2}$ \\
\hline $\mathrm{f}_{\mathrm{c} 0}$ & sem transformação & 2 & Linear & $\mathrm{f}_{\mathrm{c} 0}=\mathrm{a}+\mathrm{b} \rho_{\mathrm{bas}}$ \\
\hline $\mathrm{E}_{\mathrm{c} 90}$ & $\log E_{c 90}$ & 1 & Quadrática & $\log E_{c 90}=a+b \rho_{\text {bas }}+c \rho_{b a s}^{2}$ \\
\hline $\mathrm{f}_{\mathrm{H} 0}$ & $\sqrt{\mathrm{f}_{\mathrm{H} 0}}$ & 1 & Cúbica & $\sqrt{\mathrm{f}_{\mathrm{H} 0}}=\mathrm{a}+\mathrm{b} \rho_{\mathrm{bas}}+\mathrm{c} \rho_{\mathrm{bas}}{ }^{2}+\mathrm{d} \rho_{\mathrm{bas}}{ }^{3}$ \\
\hline $\mathrm{f}_{\mathrm{H} 90}$ & $\sqrt{\mathrm{f}_{\mathrm{H} 90}}$ & 1 & Cúbica & $\sqrt{\mathrm{f}_{\mathrm{H} 90}}=\mathrm{a}+\mathrm{b} \rho_{\mathrm{bas}}+\mathrm{c} \rho_{\mathrm{bas}}{ }^{2}+\mathrm{d} \rho_{\mathrm{bas}}{ }^{3}$ \\
\hline $\mathrm{f}_{\mathrm{w} t 90}$ & $\log f_{w t 90}$ & 0 & Quadrática & $\log f_{w t 90}=a+b \rho_{\text {bas }}+c \rho_{\text {bas }}^{2}$ \\
\hline $\mathrm{f}_{\mathrm{v} 0}$ & $\log f_{v 0}$ & 0 & Quadrática & $\log f_{v 0}=a+b \rho_{\text {bas }}+c \rho_{\text {bas }}^{2}$ \\
\hline $\mathrm{f}_{\mathrm{s} 0}$ & $\sqrt{f_{s 0}}$ & 1 & Quadrática & $\sqrt{\mathrm{f}_{\mathrm{s} 0}}=\mathrm{a}+\mathrm{b} \rho_{\mathrm{bas}}+\mathrm{c} \rho_{\mathrm{bas}}^{2}$ \\
\hline
\end{tabular}

$\rho_{\text {bas }}$ : densidade básica, em g.cm ${ }^{-3} ; \varepsilon_{\mathrm{r}, 3}$ : contração tangencial, em $\% ; \varepsilon_{\mathrm{r}, 2}$ : contração radial, em \%; $\mathrm{f}_{\mathrm{M}}$ : módulo de ruptura à flexão estática, em $\mathrm{MPa} ; \mathrm{E}_{\mathrm{M} 0}$ : módulo de elasticidade à flexão estática, em $\mathrm{MPa} ; \mathrm{f}_{\mathrm{c} 0}$ : resistência à compressão paralela às fibras, em $\mathrm{MPa}$; $\mathrm{E}_{\mathrm{c} 90}$ : resistência no limite proporcional à compressão perpendicular às fibras, em $\mathrm{MPa} ; \mathrm{f}_{\mathrm{H} 0}$ : dureza Janka paralela às fibras, em $\mathrm{N}$; $\mathrm{f}_{\mathrm{H} 90}$ : dureza Janka transversal às fibras, em $\mathrm{N} ; \mathrm{f}_{\mathrm{w}+90}$ : resistência à tração perpendicular às fibras, em $\mathrm{MPa}$; $\mathrm{f}_{\mathrm{v} 0}$ : resistência ao fendilhamento, em MPa; $\mathrm{f}_{\mathrm{s} 0}$ : resistência ao cisalhamento, em MPa; a, b, c e d: coeficientes de regressão das equações; Log: logaritmo de base 10 .

As modificações propostas pelo procedimento Guided Data Analysis promoveram um ganho substancial no ajuste das equações de regressão, o que as torna melhores quanto às estimativas. Para efeito de comparação, efetuaram-se, com os mesmos dados, os cálculos de regressão linear simples (modelo genérico y = a + bx), ou seja, sem as correções das imperfeições estatísticas prescritas pelo SAS. A tabela 7 apresenta os ganhos nos coeficientes de determinação $\left(\mathrm{R}^{2}\right)$ da regressão guiada do $\mathrm{SAS}$ em relação à regressão linear simples.

Tabela 7. Comparativo dos coeficientes de determinação $\left(\mathrm{R}^{2}\right)$ obtidos por análises de regressão linear simples com os obtidos por análises de regressão utilizando o SAS Guided Data Analysis.

Table 7. Comparative of the coefficients of determination $\left(\mathrm{R}^{2}\right)$ obtained by analysis of simple linear regression with those obtained by the regression analysis with the SAS Guided Dates Analysis.

\begin{tabular}{lcccc}
\hline Propriedade & $\begin{array}{c}\mathbf{R}^{2} \\
\text { Linear simples }\end{array}$ & $\begin{array}{c}\mathbf{R}^{2} \\
\text { SAS Guided Data Analysis }\end{array}$ & $\begin{array}{c}\text { Ganho } \\
\text { absoluto }\end{array}$ & $\begin{array}{c}\text { Ganho } \\
\text { percentual }\end{array}$ \\
\hline$\varepsilon_{\mathrm{r}, 3}$ & 0,132 & 0,132 & - & - \\
$\varepsilon_{\mathrm{r}, 2}$ & 0,183 & 0,220 & 0,037 & 20,2 \\
$\mathrm{f}_{\mathrm{M}}$ & 0,851 & 0,902 & 0,051 & 6,0 \\
$\mathrm{E}_{\mathrm{M} 0}$ & 0,795 & 0,810 & 0,015 & 1,9 \\
$\mathrm{f}_{\mathrm{c} 0}$ & 0,887 & 0,907 & 0,020 & 2,3 \\
$\mathrm{E}_{\mathrm{c} 90}$ & 0,832 & 0,889 & 0,057 & 6,9 \\
$\mathrm{f}_{\mathrm{H} 0}$ & 0,769 & 0,814 & 0,045 & 5,9 \\
$\mathrm{f}_{\mathrm{H} 90}$ & 0,848 & 0,894 & 0,046 & 5,4 \\
$\mathrm{f}_{\mathrm{w}+90}$ & 0,259 & 0,366 & 0,107 & 41,3 \\
$\mathrm{f}_{\mathrm{v} 0}$ & 0,374 & 0,477 & 0,103 & 27,5 \\
$\mathrm{f}_{\mathrm{s} 0}$ & 0,778 & 0,814 & 0,036 & 4,6 \\
\hline
\end{tabular}

$\rho_{\text {bas: }}$ : densidade básica, em g.cm ${ }^{-3} ; \varepsilon_{\mathrm{r}, 3}$ : contração tangencial, em $\% ; \varepsilon_{\mathrm{r}, 2}$ : contração radial, em $\% ; \mathrm{f}_{\mathrm{M}}$ : módulo de ruptura à flexão estática, em $\mathrm{MPa} ; \mathrm{E}_{\mathrm{M} 0}$ : módulo de elasticidade à flexão estática, em $\mathrm{MPa} ; \mathrm{f}_{\mathrm{c} 0}$ : resistência à compressão paralela às fibras, em $\mathrm{MPa}$; $\mathrm{E}_{\mathrm{c} 90}$ : resistência no limite proporcional à compressão perpendicular às fibras, em $\mathrm{MPa} ; \mathrm{f}_{\mathrm{H} 0}$ : dureza Janka paralela às fibras, em $\mathrm{N}$; $\mathrm{f}_{\mathrm{H} 90}$ : dureza Janka transversal às fibras, em $\mathrm{N} ; \mathrm{f}_{\mathrm{w} t 90}$ : resistência à tração perpendicular às fibras, em $\mathrm{MPa}$; $\mathrm{f}_{\mathrm{v} 0}$ : resistência ao fendilhamento, em MPa; $\mathrm{f}_{\mathrm{s} 0}$ : resistência ao cisalhamento, em MPa. 
O ganho médio absoluto para as 10 PFMM que sofreram modificações foi de 0,052 , cerca de 7,9\%. Em termos individuais, os ganhos mais expressivos foram das propriedades $f_{w t 90}, f_{v 0}$ e $\varepsilon_{r, 2}$, que tiveram seus $\mathrm{R}^{2}$ aumentados em $41,3 \%, 27,5 \%$ e $20,2 \%$, respectivamente.

As equações ajustadas com seus respectivos coeficientes de regressão (da equação) e de determinação $\left(\mathrm{R}^{2}\right)$, bem como o número de observações (após a eliminação dos outliers) que geraram cada equação, são apresentadas na tabela 8.

Tabela 8. Equações de regressão ajustadas para estimativas das propriedades físicas e mecânicas das madeiras (PFMM) utilizando a densidade básica $\left(\rho_{\text {bas }}\right)$ como variável independente.

Table 8. Adjusted regression equations for estimates of the physical and mechanical properties of wood (PFMM) using the basic density $\left(\rho_{\text {bas }}\right)$ as independent variable.

\begin{tabular}{lllc}
\hline Propriedade & \multicolumn{1}{c}{ Equação ajustada ** } & $\mathbf{R}^{\mathbf{2}}$ & $\mathbf{n}$ \\
\hline$\varepsilon_{\mathrm{r}, 3}$ & $\varepsilon_{\mathrm{r}, 3}=6,4526+3,6368 \rho_{\text {bas }}$ & 0,132 & 138 \\
$\varepsilon_{\mathrm{r}, 2}$ & $\varepsilon_{\mathrm{r}, 2}=12,021-43,633 \rho_{\text {bas }}+76,073 \rho_{\text {bas }}{ }^{2}-39,532 \rho_{\text {bas }}{ }^{3}$ & 0,220 & 138 \\
$\mathrm{f}_{\mathrm{M}}$ & $\sqrt{\mathrm{f}_{\mathrm{M}}}=1,4169+19,844 \rho_{\text {bas }}-7,517 \rho_{\text {bas }}$ & 0,902 & 156 \\
$\mathrm{E}_{\mathrm{M} 0}$ & $\mathrm{E}_{\mathrm{M} 0}=-1741,8+32414 \rho_{\text {bas }}-12889 \rho_{\text {bas }}{ }^{2}$ & 0,810 & 159 \\
$\mathrm{f}_{\mathrm{c} 0}$ & $\mathrm{f}_{\mathrm{c} 0}=-5,1558+108,55 \rho_{\text {bas }}$ & 0,907 & 157 \\
$\mathrm{E}_{\mathrm{c} 90}$ & $\log \mathrm{E}_{\mathrm{c} 90}=-0,1655+2,3153 \rho_{\text {bas }}-0,7795 \rho_{\text {bas }}{ }^{2}$ & 0,889 & 153 \\
$\mathrm{f}_{\mathrm{H} 0}$ & $\sqrt{\mathrm{f}_{\mathrm{H} 0}}=68,329-191,0 \rho_{\text {bas }}+553,09 \rho_{\text {bas }}{ }^{2}-306,32 \rho_{\text {bas }}{ }^{3}$ & 0,814 & 157 \\
$\mathrm{f}_{\mathrm{H} 90}$ & $\sqrt{\mathrm{f}_{\mathrm{H} 90}}=44,812-129,76 \rho_{\text {bas }}+454,44 \rho_{\text {bas }}{ }^{2}-239,73 \rho_{\text {bas }}{ }^{3}$ & 0,894 & 156 \\
$\mathrm{f}_{\mathrm{w} t 90}$ & $\log \mathrm{f}_{\mathrm{w} t 90}=-0,139+2,0218 \rho_{\text {bas }}-1,2795 \rho_{\text {bas }}{ }^{2}$ & 0,366 & 154 \\
$\mathrm{f}_{\mathrm{v} 0}$ & $\log \mathrm{f}_{\mathrm{v} 0}=-0,0103+1,9425 \rho_{\text {bas }}-1,1534 \rho_{\text {bas }}{ }^{2}$ & 0,477 & 115 \\
$\mathrm{f}_{\mathrm{s} 0}$ & $\sqrt{\mathrm{f}_{\mathrm{s} 0}}=0,7111+5,7022 \rho_{\text {bas }}-1,7949 \rho_{\text {bas }}{ }^{2}$ & 0,814 & 156 \\
\hline
\end{tabular}

$\rho_{\text {bas }}$ : densidade básica, em g.cm ${ }^{-3} ; \varepsilon_{\mathrm{r}, 3}$ : contração tangencial, em $\% ; \varepsilon_{\mathrm{r}, 2}$ : contração radial, em \%; $\mathrm{f}_{\mathrm{M}}$ : módulo de ruptura à flexão estática, em $\mathrm{MPa} ; \mathrm{E}_{\mathrm{M} 0}$ : módulo de elasticidade à flexão estática, em $\mathrm{MPa} ; \mathrm{f}_{\mathrm{c} 0}$ : resistência à compressão paralela às fibras, em $\mathrm{MPa}$; $\mathrm{E}_{\mathrm{c} 90}$ : resistência no limite proporcional à compressão perpendicular às fibras, em $\mathrm{MPa} ; \mathrm{f}_{\mathrm{H} 0}$ : dureza Janka paralela às fibras, em $\mathrm{N}$; $\mathrm{f}_{\mathrm{H} 90}$ : dureza Janka transversal às fibras, em $\mathrm{N} ; \mathrm{f}_{\mathrm{w} t 90}$ : resistência à tração perpendicular às fibras, em $\mathrm{MPa} ; \mathrm{f}_{\mathrm{v} 0}$ : resistência ao fendilhamento, em MPa; $\mathrm{f}_{\mathrm{s} 0}$ : resistência ao cisalhamento, em $\mathrm{MPa}$; **: todos os modelos apresentaram, pelo teste $\mathrm{F}$, significância ao nível de $1 \%$ de probabilidade $\left(\mathrm{P}<0,01\right.$ de Ho); $\mathrm{R}^{2}$ : coeficiente de determinação; n: número de pares de dados (observações) que gerou a equação; Log: logaritmo de base 10.

Como era de se esperar, o ranking, ou hierarquia, dos $\mathrm{R}^{2}$ das equações ajustadas foi praticamente o mesmo dos coeficientes de correlação linear $(\alpha)$ de $\rho_{\text {bas }}$ com as demais propriedades (apresentados na Tabela 5). As mudanças de posição ocorridas foram somente de $\mathrm{E}_{\mathrm{M} 0}$ e $\mathrm{f}_{\mathrm{H} 0}$, que inverteram a quinta e sétima posições, do ranking de $\alpha$ para o de $\mathrm{R}^{2}$, respectivamente.

As mudanças de posições hierárquicas e também do valor de $\mathrm{R}^{2}$, em relação aos $\alpha$ de $\rho_{\text {bas}}$, uma vez que não houve correspondência quadrática entre esses coeficientes, estão relacionados às alterações promovidas pelas análises das suposições estatísticas do programa SAS. Isso é comprovado pelos resultados da relação funcional de $\varepsilon_{\mathrm{r}, 3} \operatorname{com} \rho_{\text {bas }}$, pois foi o único caso em que a análise guiada do SAS não indicou qualquer alteração e, assim, a relação entre $\mathrm{R}^{2}$ e $\alpha$ foi a esperada $\left(\sqrt{\mathrm{R}^{2}}\right.$ de $\varepsilon_{\mathrm{r}, 3}=\alpha$ de $\varepsilon_{\mathrm{r}, 3} \therefore$ $\sqrt{0,132}=0,363)$.

A maioria das equações de regressão apresentou um $\mathrm{R}^{2}$ que pode ser considerado satisfatório, ou seja, acima de 0,8 . O maior valor encontrado foi o da equação para estimar $\mathrm{f}_{\mathrm{c} 0}\left(\mathrm{R}^{2}=0,907\right)$, e o menor valor foi o da equação para estimar $\varepsilon_{\mathrm{r}, 3}\left(\mathrm{R}^{2}=0,132\right)$, significando, respectivamente, o melhor e o pior desempenho dessas equações quanto à precisão de estimativas.

Como eram esperadas, as dependências funcionais de $\rho_{\text {bas }}$ com as propriedades físicas de contração $\left(\varepsilon_{\mathrm{r}, 3}\right.$ e $\left.\varepsilon_{\mathrm{r}, 2}\right)$ foram as mais baixas entre todas, e as com as propriedades mecânicas $f_{\mathrm{w} t 90}$ e $\mathrm{f}_{\mathrm{v} 0}$ pouco significativas. Assim, as equações de $\rho_{\mathrm{bas}} \operatorname{com} \varepsilon_{\mathrm{r}, 3}, \varepsilon_{\mathrm{r}, 2}, \mathrm{f}_{\mathrm{wt} 90}$ e $\mathrm{f}_{\mathrm{v} 0}$ podem ser consideradas inviáveis para efeito de estimativas.

Quanto ao número de observações, ou pares de dados, usados para o ajuste de cada equação, houve variação entre um máximo de 159 para $\mathrm{E}_{\mathrm{M} 0}$, e um mínimo de 115 para $\mathrm{f}_{\mathrm{v} 0}$. Tal variação deveu-se à ausência de alguns dados nas fontes de consulta e à eliminação dos outliers.

Com relação aos outliers, foram seis os conjuntos de dados em que ocorreram. Dois conjuntos $\left(f_{M}\right.$ e $\left.f_{c 0}\right)$ tiveram a exclusão de duas observações, e quatro $\left(E_{c 90}, f_{H 0}, f_{H 90}\right.$ e $\left.f_{s 0}\right)$ tiveram a exclusão de uma 
observação. Em geral, a ocorrência de outliers se deu nos conjuntos de dados que apresentaram os maiores coeficientes de variação percentual $(\mathrm{Cv} \%)$, concluindo-se que contribuíram para o valor desse coeficiente.

As figuras 2 e 3 exibem a dispersão dos dados e linhas de tendência das equações de regressão ajustadas. Em algumas situações (a exemplo das curvas de $\rho_{\text {bas }}$ com $f_{M}$ e $E_{M 0}$ ), a forma da dispersão gráfica dos dados mostra claramente que é justificada a indicação do modelo da equação (linear, quadrática ou cúbica) proposta pela análise guiada do programa SAS.
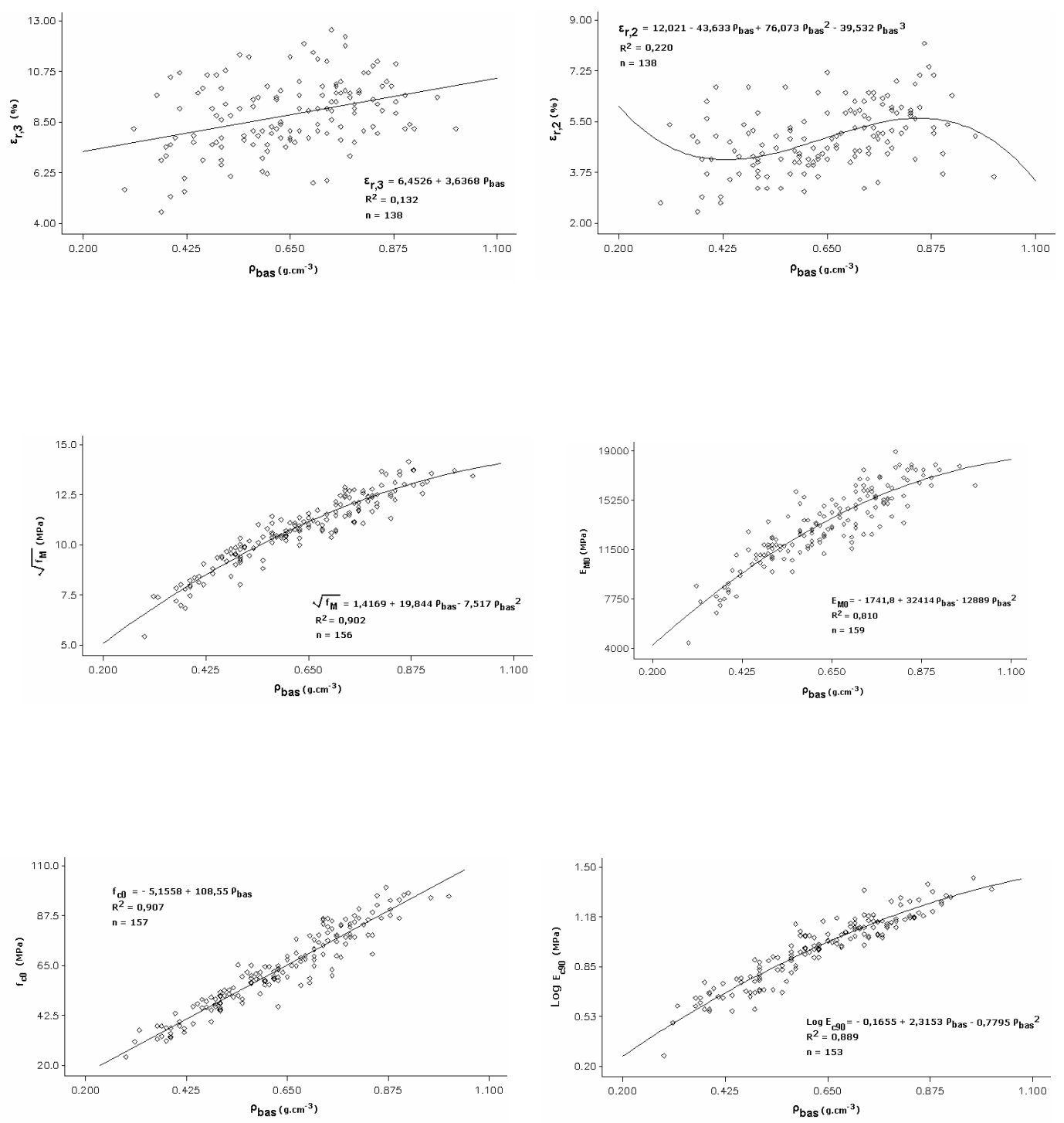

Figura 2. Dispersão dos dados e linha de tendência das equações de regressão para as propriedades $\varepsilon_{\mathrm{r}, 3}$ (contração tangencial), $\varepsilon_{\mathrm{r}, 2}$ (contração radial), $\mathrm{f}_{\mathrm{M}}$ (módulo de ruptura à flexão estática), $\mathrm{E}_{\mathrm{M} 0}$ (módulo de elasticidade à flexão estática), $\mathrm{f}_{\mathrm{c} 0}$ (resistência à compressão paralela às fibras) e $\mathrm{E}_{\mathrm{c} 90}$ (resistência no limite proporcional à compressão perpendicular às fibras) em função da $\rho_{\mathrm{bas}}$ (densidade básica).

Figure 2. Dispersion of the data and tendency line of the regression equations for the properties $\varepsilon_{\mathrm{r}, 3}$ (tangential shrinkage), $\varepsilon_{\mathrm{r}, 2}$ (radial shrinkage), $\mathrm{f}_{\mathrm{M}}$ (rupture module to the static bending), $\mathrm{E}_{\mathrm{M} 0}$ (module of elasticity to the static bending), $\mathrm{f}_{\mathrm{c} 0}$ (resistance to the parallel compression to the fibers) and $\mathrm{E}_{\mathrm{c} 90}$ (resistance in the proportional limit to the perpendicular compression to the fibers) in function of $\rho_{\text {bas }}$ (basic density). 

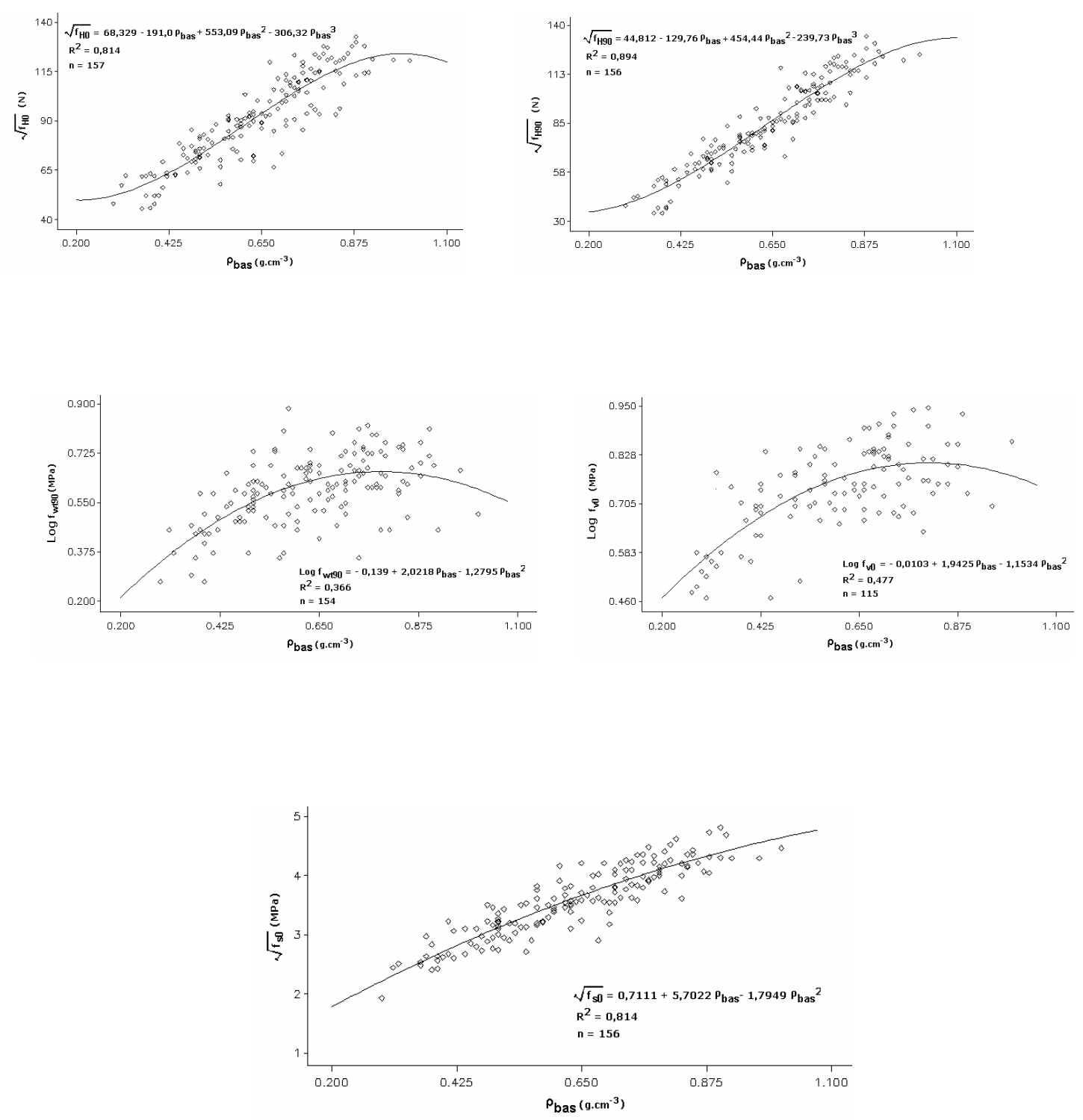

Figura 3. Dispersão dos dados e linha de tendência das equações de regressão para as propriedades $\mathrm{f}_{\mathrm{H} 0}$ (dureza Janka paralela às fibras), $\mathrm{f}_{\mathrm{H} 90}$ (dureza Janka transversal às fibras), $\mathrm{f}_{\mathrm{w} t 90}$ (resistência à tração perpendicular às fibras), $\mathrm{f}_{\mathrm{v} 0}$ (resistência ao fendilhamento) e $\mathrm{f}_{\mathrm{s} 0}$ (resistência ao cisalhamento) em função da $\rho_{\text {bas }}$ (densidade básica).

Figure 3. Dispersion of the data and tendency line of the regression equations for the properties $\mathrm{f}_{\mathrm{H} 0}$ (Janka hardness parallel to the grain), $\mathrm{f}_{\mathrm{H} 90}$ (Janka hardness crossection to the grain), $\mathrm{f}_{\mathrm{w}+90}$ (resistance to the perpendicular traction to the fibers), $f_{v 0}$ (resistance to the cleavage) and $f_{s 0}$ (resistance to the shear) in function of $\rho_{\text {bas }}$ (basic density).

\section{CONCLUSÕES}

Para as propriedades que apresentaram altos valores nas suas relações funcionais $\left(\alpha\right.$ e $\left.R^{2}\right)$, ou seja, $\rho_{\text {bas }}, \mathrm{f}_{\mathrm{M}}, \mathrm{E}_{\mathrm{M} 0}, \mathrm{f}_{\mathrm{c} 0}, \mathrm{E}_{\mathrm{c} 90}, \mathrm{f}_{\mathrm{H} 0}, \mathrm{f}_{\mathrm{H} 90} \mathrm{e} \mathrm{f}_{\mathrm{s} 0}$, as análises de regressão possibilitaram a obtenção de equações satisfatórias para fins de estimativas dessas propriedades, sobretudo para a determinação das PFMM de espécies madeireiras brasileiras ainda não ensaiadas. 
Por outro lado, os baixos valores de $\alpha$ e $\mathrm{R}^{2}$ das propriedades de contração $\left(\varepsilon_{\mathrm{r}, 3}\right.$ e $\left.\varepsilon_{\mathrm{r}, 2}\right)$, bem como das propriedades de resistência a forças de tração $\left(f_{w t 90}\right)$ e fendilhamento $\left(f_{v 0}\right)$, inviabilizam vínculos matemáticos correlatos com a maioria das outras propriedades e indicam que estas são influenciadas predominantemente por outros fatores, afora a quantidade do material lenhoso da madeira ( $\left.\rho_{\text {bas }}\right)$, a exemplo dos relacionados à anatomia e à presença de extrativos.

\section{AGRADECIMENTO}

Ao Centro de Pesquisa Agroflorestal do Acre (CPAF-AC), uma das unidades amazônicas da Empresa Brasileira de Pesquisa Agropecuária (EMBRAPA), pela oportunidade de realização deste trabalho e pelo apoio financeiro concedido.

\section{REFERÊNCIAS}

ARAÚJO, H. J. B. Agrupamento das espécies madeireiras ocorrentes em pequenas áreas sob manejo florestal do Projeto de Colonização Pedro Peixoto (AC) por similaridade das propriedades físicas e mecânicas. $168 \mathrm{f}$. Dissertação (Mestrado em Recursos florestais) - Escola Superior de Agricultura "Luiz de Queiroz", Piracicaba, 2002.

ARGANBRIGHT, D. G. Influence of extractives on bending strenght of redwood (Sequoia sempervirens). Wood and Fiber, Madison, v. 2, n. 4, 367-372, 1971.

ASSOCIAÇÃO BRASILEIRA DE NORMAS TÉCNICAS. NBR 7190: Projeto de estruturas de madeira. Rio de Janeiro, 1997. 107 p.

BODIG, J.; JAYNE, B. A. Mechanics of wood and wood composites. New York: Van Nostrand Reinhold, 1982. 712 p.

BROWN, H. P; PANSHIN, A. J.; FORSAITH, C. C. Textbook of wood technology: structure, identification, defects, and uses of the commercial wood of the Inited Atates. New York: McGraw-Hill, 1949. v. 1. 652 p.

CHIMELO, J. P. Development of a probability-based computerization system for identification and for property prediction of selected tropical hardwoods. $202 \mathrm{f}$. Thesis - Virginia Polytechnic Institute, Blacksburg, 1980.

EVANS, J. L. W.; SENFT, J. F.; GREEN, D. W. Juvenile wood effect in red alder: analysis of physical and mechanical data to delineate juvenileand mature wood zones. Forest Products Journal, Madison, v. 50, n. 7/8, p. 75-87, 2000.

FOREST PRODUCTS LABORATORY. Wood handbook: wood as an engeneering material. Madison: USDA, 1974. $1 \mathrm{v}$.

GARCIA, J. N.; QUIRINO, W. F. Influência dos extrativos solúveis em água na resistência à compressão paralela da madeira de ipê (Tabebuia sp.). In: Congresso Florestal Pan-Americano, 1., 1993, Curitiba.; Congresso Florestal Brasileiro, 7., 1993, Curitiba. Anais.... Curitiba, 1993. 2 v. p. 647-650.

IBAMA. Madeiras da Amazônia: características e utilização. v. 3. Amazônia Oriental. Brasília, DF, 1997. $141 \mathrm{p}$.

INSTITUTO BRASILEIRO DE DESENVOLVIMENTO FLORESTAL. Madeiras da Amazônia: características e utilização: Floresta Nacional do Tapajós. Brasília, DF, 1981. v. 1. 113 p.

INSTITUTO BRASILEIRO DE DESENVOLVIMENTO FLORESTAL. Madeiras da Amazônia: características e utilização: Estação Experimental de Curuá-Una. Brasília, DF, 1988. v. 2. 236 p.

JANKOWSKY, I. P. Influência da densidade básica e do teor de extrativos na umidade de equilíbrio da madeira. 94 f. Dissertação (Mestrado) - Escola Superior de Agricultura "Luiz de Queiroz", Piracicaba, 1979. 
KOLLMANN, F. E. P.; CÔTÉ JUNIOR, W. A. Principles of wood science and technology: solid wood. Berlin: Spring-Verlag; 1968. 592 p.

LOPES, G. A.; GARCIA, J. N. Densidade básica e umidade natural da madeira de Eucalyptus saligna Smith, de Itatinga, associadas aos padrões de casca apresentados pela população. Scientia Forestalis, Piracicaba, n. 62, p. 13-23, 2002.

NASCIMENTO, C. C.; GARCIA, J. N.; DIÁZ, M. P. Agrupamento de espécies madeireiras da Amazônia em função da densidade básica e propriedades mecânicas. Madera y Bosques, Veracruz, v. 3, n. 1, p. 33$52,1997$.

NAHUZ, M. A. R. Some aspects of the introduction of lesser-known brazilian species to the European timber market. Thesis (Magister) -, Department of Forestry and Wood Science, University College of North Wales, Bangor, 1974.

NASCIMENTO, C. C. Variabilidade da densidade básica e de propriedades mecânicas de madeiras da Amazônia. 129 f. Tese (Mestrado) - Escola Superior de Agricultura "Luiz de Queiroz", Piracicaba, 1993.

PANSHIN, A. J.; ZEEUW, C. Textbook of wood technology. 3. ed., New York: Mcgraw-Hill, 1970. v. $1.705 \mathrm{p}$.

PAUlA, E. V. C. M.; CABRAL, M. T. F. D.; NASCIMENTO, C. C.; ROCHA, J. S. Propriedades mecânicas de trinta e nove espécies de madeiras do Amazonas. In: ENCONTRO BRASILEIRO EM MADEIRAS EEM ESTRUTURAS DE MADEIRA, 2., 1986, São Paulo. Anais... São Paulo, 1986. 1 v.

RICHTER, H. G.; BURGER, L. M. Anatomia da Madeira. 2. ed. Curitiba: Universidade Federal do Paraná, 1978. 78 p.

ROCHA, J. S.; PAULA, E. V. C. M.; SIQUEIRA, M. L. Flexão estática em amostras pequenas livres de defeitos. Acta Amazônica, Manuas, v. 18, n. 1/2, p. 147-162, 1988.

ROCHA, J. S. A segurança de estruturas de madeira determinada a partir da variabilidade da densidade básica e de propriedades mecânicas de madeiras amazônicas. 160 f. Dissertação (Mestrado) - Escola Superior de Agricultura "Luiz de Queiroz", Piracicaba, 1994.

SAS INSTITUTE INC. SAS/STAT: User's Guide: Version 6. 2. ed. Cary, 1993. 1022 p.

SIAU, J. F. Transport processes in wood. Berlim: Spring-Verlag, 1984. 223 p.

SOUZA, M. H.; MAGLIANO, M. M.; CAMARGOS, J. A. A.; SOUZA, M. R. Madeiras tropicais brasileiras. Brasília, DF: IBAMA, 1997. 152 p.

USDA. Wood handbook: wood as engineering material. Washington, DC: U.S. Department of Agriculture. Forest Service, 1999. 473 p. (Agriculture Handbook, n. 72).

WANGAARD, F. F. The mechanical properties of wood. New York: J. Wiley, 1950. 377 p. 